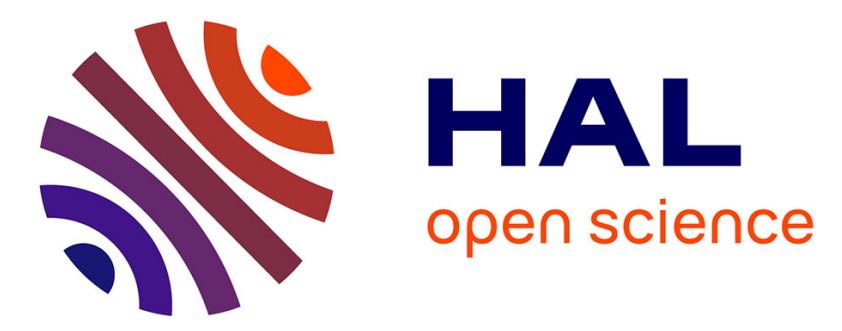

\title{
KEWPIE2: A cascade code for the study of dynamical decay of excited nuclei
}

H. Lu, A. Marchix, Y. Abe, D. Boilley

\section{To cite this version:}

H. Lu, A. Marchix, Y. Abe, D. Boilley. KEWPIE2: A cascade code for the study of dynamical decay of excited nuclei. Computer Physics Communications, 2016, 200, pp.381-399. 10.1016/j.cpc.2015.12.003 . in2p3-01214344

HAL Id: in2p3-01214344

https://hal.in2p3.fr/in2p3-01214344

Submitted on 12 Oct 2015

HAL is a multi-disciplinary open access archive for the deposit and dissemination of scientific research documents, whether they are published or not. The documents may come from teaching and research institutions in France or abroad, or from public or private research centers.
L'archive ouverte pluridisciplinaire HAL, est destinée au dépôt et à la diffusion de documents scientifiques de niveau recherche, publiés ou non, émanant des établissements d'enseignement et de recherche français ou étrangers, des laboratoires publics ou privés. 


\title{
KEWPIE2: A Cascade Code for the Study of Dynamical Decay of Excited Nuclei
}

\author{
Hongliang L L̈̈ ${ }^{\mathrm{a}, \mathrm{b}}$, Anthony Marchix ${ }^{\mathrm{a}, \mathrm{b}, \mathrm{d}, 1, *,}$, Yasuhisa Abe ${ }^{\mathrm{c}}$, David Boilley $\mathrm{y}^{\mathrm{a}, \mathrm{b}, * *}$ \\ ${ }^{a}$ GANIL, CEA/DSM-CNRS/IN2P3, BP 55027, F-14076 Caen cedex 5, France \\ ${ }^{b}$ Normandie Université, France \\ ${ }^{c}$ RCNP, Osaka University, Ibaraki, Osaka 567-0047, Japan \\ ${ }^{d}$ CEA, Centre de Saclay, Irfu/SPhN, F-91191 Gif-sur-Yvette, France
}

\begin{abstract}
KEWPIE - a cascade code devoted to investigating the dynamical decay of excited nuclei, specially designed for treating very low probability events related to the synthesis of super-heavy nuclei formed in fusion-evaporation reactions - has been improved and rewritten in $\mathrm{C}++$ programing language to become KEWPIE2. The current version of the code comprises various nuclear models concerning the light-particle emission, fission process and statistical properties of excited nuclei. General features of the code, such as the numerical scheme and the main physical ingredients, are described in detail. Some typical calculations having been performed in the present paper clearly show that theoretical predictions are generally in accordance with experimental data. Furthermore, since the values of some input parameters cannot be determined neither theoretically nor experimentally, a sensibility analysis is presented. To this end, we systematically investigate the effects of using different parameter values and reaction models on the final results. As expected, in the case of heavy nuclei, the fission process has the most crucial role to play in theoretical predictions. This work would be essential for numerical modeling of fusion-evaporation reactions.
\end{abstract}

Keywords: Fusion-evaporation reaction, Bateman equations, Dynamical cascade, Fission time

\section{Introduction}

The KEWPIE code was designed to investigate the radioactive decay of excited heavy and super-heavy nuclei formed in fusion-evaporation reactions. It refers to a dynamical cascade code that avoids Monte-Carlo methods so as to increase computational efficiency when dealing with extremely low probability events. The numerical scheme is essentially based on the Bateman equations, which enable us to compute both statistical and dynamical observables, such as the survival probability of a decaying nucleus and the fission-time distribution. The latter was initially motivated by the experimental measurements carried out at the GANIL (Grand Accélérateur National d'Ions Lourds) laboratory [1-4].

The first version of KEWPIE [5] was released in 2004. KEWPIE2 is an upgraded version of the KEWPIE code, which was completely rewritten in $\mathrm{C}++$ language [6]. Accordingly, owing to the object-oriented features of $\mathrm{C}++$, further developments would become much easier. The code has been continually developed since that time and was employed to provide theoretical predictions for many applications [7-10]. In the current version, the basic algorithm was modified and the main physical contents have been greatly improved by incorporating various theoretical models. As a consequence, some parameter values that appeared incorrect have been corrected and thus become

\footnotetext{
${ }^{*}$ Principal corresponding author

** Corresponding author

Email addresses: anthony.marchix@cea.fr (Anthony Marchix ), david.boilley@ganil.fr (David Boilley)
}

more consistent with recent advances in nuclear physics with heavy ions.

The fusion-evaporation reaction is of special interest within many areas of nuclear physics. It is commonly used to synthesize exotic nuclei far from the valley of stability and thus to explore the boundaries of the nuclide chart. To illustrate it, one might consider the following process: the collision of a projectile, $a$, with a target nucleus, $A$, forms an intermediate state, $C$, called compound nucleus, which subsequently evaporates a light particle $b$, thus transforming itself into a residual nucleus $B$. The whole process can be illustrated as follows:

$$
a+A \longrightarrow C^{*} \longrightarrow B+b
$$

where the compound nucleus is denoted by $C$ and the asterisk indicates an excited state characterized by nuclear temperature $T$. In general, such a reaction can be divided into two stages: the collision of two nuclei leading to the formation of a compound nucleus and its subsequent decay by light-particle evaporation in competition with nuclear fission and $\gamma$-ray emission. Based upon the so-called Bohr independence hypothesis [11], these two reaction steps are considered independent of each other. Accordingly, the evaporation-residue (ER) crosssection can thus be expressed as

$$
\sigma_{\mathrm{ER}}\left(E_{\mathrm{cm}}\right)=\sum_{J_{C} \geq 0} \sigma_{\text {fus }}\left(E_{\mathrm{cm}}, J_{C}\right) P_{\text {surv }}\left(E_{C}^{*}, J_{C}\right),
$$

with

$$
\sigma_{\text {fus }}\left(E_{\mathrm{cm}}, J_{C}\right)=\frac{\pi}{k^{2}}\left(2 J_{C}+1\right) P_{\text {fus }}\left(E_{\mathrm{cm}}, J_{C}\right)
$$


where $J_{C}$ represents the total angular momentum of the compound system and $k$ the wave number of relative motion between the projectile and target nuclei. In writing Eqs. (1) and (2), we have employed the No-Coriolis or Iso-centrifugal approximation [12], which states that the entrance-channel orbital angular momentum of the binary system can be replaced by the total angular momentum or the spin of the compound nucleus. The relationship between the incident energy in the centre-of-mass frame $E_{\mathrm{cm}}$ and the total excitation energy of the compound nucleus $E_{C}^{*}$ is simply given by $E_{C}^{*}=E_{\mathrm{cm}}+Q$, where $Q$ is evaluated from the latest measured ground-state masses $[13,14]$. If the experimental values are not available, the theoretical mass table [15] will be employed.

The KEWPIE2 code mainly handles the final stage of the fusion-evaporation reaction. The physical processes included in the code remain basically unchanged compared to the previous version. As a whole, they are divided into two main categories, namely light-particle evaporation and nuclear fission. To describe the emission of light particles, the code has accommodated two standard approaches, namely the WeisskopfEwing [16, 17] and Hauser-Feshbach models [18]. It should be noted that the latter takes into account the spins of the mother and daughter nuclei, whereas the former can be somewhat regarded as a classical limit that neglects the intrinsic quantum states of the compound system. Owing to its simplicity, the Weisskopf-Ewing evaporation model has been commonly employed in analytical calculations on the synthesis of superheavy nuclei [19-21]. Nevertheless, as emphasized in [2231], the Hauser-Feshbach formalism appears more suitable and would be recommended for heavy-ion reaction calculations in spite of its computational inefficiency. As regards the fission process, the decay rate can be estimated within the framework of the Bohr-Wheeler statistical theory [32]. According to Kramers [33], nuclear fission can be described by a diffusion process above the potential barrier along the deformation coordinate. Since this pioneering work, it has been well established that the dynamical effect of nuclear fission can be modeled using the Klein-Kramers equation or its equivalent Langevin equation [34-38]. Accordingly, the whole process is not only dependent on the potential-energy landscape of the compound system, but also on the friction parameter characterizing the nuclear viscosity. As a result, the fission rate has to take a finite time to reach its stationary value. This transient effect has been included in the code by introducing a user-defined time delay, which would be necessary when computing some dynamical observables related to the fission rate. Moreover, the groundstate and saddle-point deformations have also been taken into consideration in our model. Such effects on the nuclear structure would be essential as pointed out in Ref. [39]. In addition to the two main processes described above, some recent developments $[40,41]$ in modeling the $\gamma$-ray emission have been considered as well.

Apart from the de-excitation process, KEWPIE2 provides a calculation of the fusion probability using either the semiclassical approximation with a proximity potential [42-45] or the empirical barrier-distribution method [19, 46]. In lightion induced reactions, the fusion probability exactly corre- sponds to the one for surmounting the Coulomb (or Bass barrier [47]), that is, to the capture probability. The physical ingredients for calculating fusion cross-sections will be presented in Section 3. Furthermore, it has been empirically known that, when the charge product of the projectile-target combination $Z_{a} Z_{A} \gtrsim 1600-1800$, the collision of heavy ions does not automatically lead to the formation of compound nuclei, even if the incident energy is higher than the top of the Coulomb barrier. This phenomenon has been well confirmed and is commonly called "fusion hindrance" [48, 49], which implies that the fusion probability is not generally given by the capture one, but requires an additional factor to describe the whole process. This is considered to be due to the very strong Coulomb repulsion between two colliding nuclei. Consequently, the di-nucleus formed by the contact of two heavy ions has an extremely large deformation and is thus located outside the conditional saddle point predicted on the basis of the liquid drop model [50-55]. Put differently, the compound system has to overcome an inner barrier to fuse together, or it undergoes the so-called quasifission process $[56,57]$. In brief, the heavy-ion fusion reaction would be composed of two successive stages, namely the capture and formation steps. Within the framework of the two-step model $[58,59]$, the total fusion probability can be written in the following form:

$$
P_{\text {fus }}=P_{\text {cap }} \cdot P_{\text {form }},
$$

where $P_{\text {form }}$ stands for the compound-nucleus formation probability. It should be mentioned that the current version of the code does not provide any calculation of the formation probability because some ambiguities still persist on a theoretical level. Therefore, an option has been added in the input file in order to accommodate other fusion models.

The aim of the present paper is to provide a complete description of the KEWPIE2 code. First, we start by recalling the numerical framework for describing a dynamical decay process and some of its simple implementations. Then, the various nuclear models currently being included in the code are presented in detail. After that, some typical calculation examples are shown and the computational results are carefully compared with the available experimental data, and in the meantime, we perform a sensibility analysis for both input parameters and reaction models. Finally, some conclusions are drawn and future prospects are discussed as well.

\section{Numerical framework for compound-nucleus decay}

This section briefly presents the conceptual framework for modeling physical processes. For the sake of completeness, we first recall some basic ideas and definitions. A detailed description of the numerical scheme is then provided.

\subsection{Single chain}

\subsubsection{Population as a function of time}

Let us consider a single cascade-decay chain starting from an excited compound nucleus, under the assumption that the competition only occurs between the neutron evaporation and 
nuclear fission. The general equations describing the time evolution of such a disintegration chain read

$$
\begin{aligned}
& \frac{\mathrm{d} P_{0}}{\mathrm{~d} t}=-\Gamma_{\text {tot }}^{0} P_{0} \quad \text { with } \quad P_{0}(0)=1, \\
& \frac{\mathrm{d} P_{1}}{\mathrm{~d} t}=\Gamma_{n}^{0} P_{0}-\Gamma_{\text {tot }}^{1} P_{1} \quad \text { with } \quad P_{2}(0)=0, \\
& \vdots \\
& \frac{\mathrm{d} P_{s}}{\mathrm{~d} t}=\Gamma_{n}^{s-1} P_{s-1}-\Gamma_{\text {tot }}^{s} P_{s} \quad \text { with } \quad P_{s}(0)=0,
\end{aligned}
$$

where $\Gamma_{\text {tot }}^{s}$ is the total decay width for the nucleus labeled with $s$ and $\Gamma_{n}^{s}$ the particle-evaporation width. The total decay width is then $\Gamma_{\text {tot }}^{s}=\Gamma_{n}^{s}+\Gamma_{f}^{s}$, where $\Gamma_{f}^{s}$ corresponds to the fission-decay width. Here, $P_{s}(t)$ denotes the population of the nucleus having emitted $s$ neutrons at time $t$. If the decay widths are timeindependent, it is very easy to solve analytically these coupleddifferential equations with the help of the Laplace transform. According to [60], the corresponding solutions can be summarized as follows:

$$
\begin{aligned}
P_{0}(t) & =e^{-\Gamma_{\mathrm{tot}}^{0} t}, \\
P_{1}(t) & =\frac{\Gamma_{n}^{0}}{\Gamma_{\text {tot }}^{1}-\Gamma_{\mathrm{tot}}^{0}}\left(e^{-\Gamma_{\mathrm{tot}}^{0} t}-e^{-\Gamma_{\mathrm{tot}}^{1} t}\right), \\
\vdots & \\
P_{s_{\max }}(t) & =\prod_{k=0}^{s_{\max }-1} \Gamma_{n}^{k} \sum_{i=0}^{s_{\max }} \frac{e^{-\Gamma_{\mathrm{tot}}^{i} t}}{\prod_{j \neq i}\left(\Gamma_{\mathrm{tot}}^{j}-\Gamma_{\mathrm{tot}}^{i}\right)},
\end{aligned}
$$

where it has been assumed that the $\Gamma$ 's are all different from each other and are not equal to zero. $s_{\max }$ is the maximum number of neutrons ejected from the compound nucleus. Once all populations have been worked out, it would not be difficult to compute some measured observables.

\subsubsection{Experimental observables}

Here, we would like to provide some typical examples that were already given in [61, 62]. The probability for the compound nucleus to emit exactly $s$ neutrons prior to fission is given by

$$
p_{s}=\int_{0}^{+\infty} \mathrm{d} t \Gamma_{f}^{s} P_{s}(t)=\frac{\Gamma_{f}^{s}}{\Gamma_{\text {tot }}^{s}} \prod_{i=0}^{s-1} \frac{\Gamma_{n}^{i}}{\Gamma_{\text {tot }}^{i}} .
$$

At the end of the cascade-decay chain, it turns into

$$
p_{s_{\max }}=\prod_{i=0}^{s_{\max }} \frac{\Gamma_{n}^{i}}{\Gamma_{\mathrm{tot}}^{i}}
$$

These results have commonly been implemented in various statistical codes. Thus, the average neutron multiplicity reads

$$
\langle v\rangle=\sum_{i=0}^{s_{\max }-1} s p_{s} .
$$

Other observables related to $p_{s}$ can also be defined in a similar manner.
Finally, we are also able to estimate the average fission time which can be evaluated within the same framework. To compute this quantity, the population of each isotope at any time must be available. It is thus given by

$$
\begin{aligned}
\tau_{f} & =-\frac{1}{P_{\text {tot }}(0)-P_{\text {tot }}(+\infty)} \int_{0}^{+\infty} t \frac{\mathrm{d} P_{\text {tot }}}{\mathrm{d} t} \mathrm{~d} t, \\
& =\frac{1}{P_{\text {tot }}(0)-P_{\text {tot }}(+\infty)} \sum_{s=0}^{s_{\max }} \int_{0}^{+\infty} t \Gamma_{f}^{s} P_{s}(t) \mathrm{d} t,
\end{aligned}
$$

where $P_{\text {tot }}(0)$ and $P_{\text {tot }}(+\infty)$ denote the total population at initial time and its long-time limit, respectively. It should be mentioned that the KEWPIE2 code can be employed to estimate not only statistical observables, but also dynamical ones, such as the survival probability and the fission-time distribution. the former corresponds to the long-time limit whereas the latter requires a full resolution of the Bateman equations.

\subsection{Multi-channel scheme}

At high excitation energies, charged particles can be emitted as well. In the KEWPIE2 code, we only consider two kinds of charged particles, namely protons and $\alpha$ particles.

The populations are labeled as $P_{i, j}$ with $i$ being the number of evaporated neutrons and $j$ that of protons. To keep a triangular form, the populations are ordered following the number of evaporated nucleons, starting with neutrons. In a more formal way, one has $r=(i+j)(i+j+1) / 2+j$, where $r$ is the rank for each nucleus present in the decay chain. Similar time-dependent differential equations including the evaporation of charged particles can thus be written down without any difficulty. The first ones are given as follows:

$$
\begin{aligned}
\frac{\mathrm{d} P_{0,0}}{\mathrm{~d} t} & =-\Gamma_{\text {tot }}^{0,0} P_{0,0} \quad \text { with } \quad P_{0,0}(0)=1, \\
\frac{\mathrm{d} P_{1,0}}{\mathrm{~d} t}= & \Gamma_{n}^{0,0} P_{0,0}-\Gamma_{\text {tot }}^{1,0} P_{1,0} \quad \text { with } \quad P_{1,0}(0)=0, \\
\frac{\mathrm{d} P_{0,1}}{\mathrm{~d} t}= & \Gamma_{p}^{0,0} P_{0,0}-\Gamma_{\text {tot }}^{0,1} P_{0,1} \quad \text { with } \quad P_{0,1}(0)=0, \\
\frac{\mathrm{d} P_{2,0}}{\mathrm{~d} t}= & \Gamma_{n}^{1,0} P_{1,0}-\Gamma_{\text {tot }}^{2,0} P_{2,0} \quad \text { with } \quad P_{2,0}(0)=0, \\
\frac{\mathrm{d} P_{1,1}}{\mathrm{~d} t}= & \Gamma_{n}^{0,1} P_{0,1}+\Gamma_{p}^{1,0} P_{1,0}-\Gamma_{\text {tot }}^{1,1} P_{1,1} \\
\frac{\mathrm{d} P_{0,2}}{\mathrm{~d} t}= & \Gamma_{p}^{0,1} P_{0,1}-\Gamma_{\text {tot }}^{0,2} P_{0,2} \quad \text { with } \quad P_{0,2}(0)=0, \\
\vdots & \\
\frac{\mathrm{d} P_{2,2}}{\mathrm{~d} t}= & \Gamma_{p}^{2,1} P_{2,1}+\Gamma_{n}^{1,2} P_{1,2}+\Gamma_{\alpha}^{0,0} P_{0,0}-\Gamma_{\text {tot }}^{2,2} P_{2,2} \\
& \text { with } \quad P_{2,2}(0)=0,
\end{aligned}
$$

Here, $\Gamma_{\text {tot }}^{i, j}$ refers to the total decay width of the residual nucleus after evaporating $i$ neutrons and $j$ protons. Other $\Gamma$ 's are the particle-evaporation and fission-decay widths, respectively, according to their subscripts. 


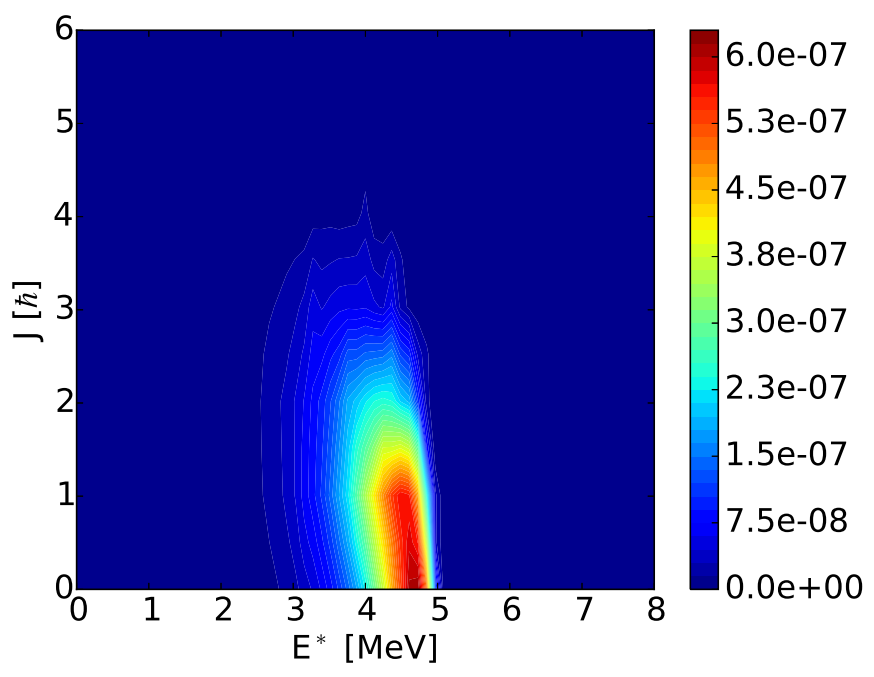

Fig. 1. Example of a two-dimensional spectrum of the daughter nucleus obtained using the spectral discretization method. The horizontal axis denotes the excitation energy and the vertical axis the nuclear spin. The color bar indicates the population distribution (in arbitrary units).

The population of each decaying nucleus corresponds to the sum of contributions of all possible decay paths. For instance, in the case of evaporation of neutrons and protons, we have $(i+j) ! /(i ! j !)$ possible paths from the initial nucleus $(0,0)$ to $(i, j)$. Using the same Laplace transform technique, one ends up with a general formula that is of course the sum of contributions of all single chains. Indeed, the linearity of the Bateman equations allows tracing of interconnected paths by manually accumulating results from separate calculations for each single chain. This general property would also be valid for calculated observables and thus makes it possible to collect similar terms together at each step. However, as the number of chains goes up, formulas will surely become much more complicated.

As mentioned in Ref. [5], the complexity of calculations is eventually due to the multi-channel scheme, but not to the dynamics that can be exactly implemented. It should be mentioned that, in the KEWPIE2 code, statistical observables are directly computed with the help of a specially-designed algorithm without exactly solving the Bateman equations.

\subsection{Numerical scheme}

The numerical implementation is briefly discussed in this subsection. For the sake of simplicity, we only focus upon the scheme for a single cascade-decay chain. It can be then extended to a multi-channel cascade decay in a similar way. One of the most essential features of the KEWPIE2 code is that the energy spectra of produced nuclei and their angular-momentum distributions (when the Hauser-Feshbach formalism is chosen) are completely calculated and processed. In our case, MonteCarlo methods might not be suitable, since we are mainly interested in a tiny fraction of events leading to the formation of heavy or super-heavy nuclei.

\subsubsection{Discretization of the population spectrum}

Supposing that one has a mother nucleus labeled with $n$. Its population spectrum $S_{n}\left(E_{n}^{*}, J_{n}\right)$, which is a function of the excitation energy $E_{n}^{*}$ and angular momentum $J_{n}$, can be discretized into $N$ and $M$ bins, respectively. The normalization condition results in

$$
\begin{aligned}
P_{n} & =\sum_{J_{n}} \int_{0}^{+\infty} S_{n}\left(E_{n}^{*}, J_{n}\right) \mathrm{d} E_{n}^{*} \\
& =\sum_{j^{\prime}}^{N} \sum_{k^{\prime}}^{M} S_{n}\left(j^{\prime}, k^{\prime}\right) .
\end{aligned}
$$

Regarding the daughter nucleus, the spectral bin $(j, k)$ corresponding to the excitation energy $E_{n+1}^{*}$ and angular momentum $J_{n+1}$ is thus fed by

$$
\delta S_{n+1}(j, k)=\sum_{J_{n}} \int_{0}^{+\infty} \mathrm{d} E_{n}^{*} S_{n}\left(E_{n}^{*}, J_{n}\right) \frac{\gamma\left(E_{n}^{*} \rightarrow E_{n+1}^{*}, J_{n}\right)}{\Gamma_{\text {tot }}^{n}}
$$

where the total decay width $\Gamma_{\text {tot }}^{n}$ has already been integrated over the whole range of excitation energies. The function $\gamma\left(E_{n}^{*} \rightarrow\right.$ $\left.E_{n+1}^{*}, J_{n}\right)$ is proportional to $\rho\left(E_{n+1}^{*}\right) / \rho\left(E_{n}^{*}\right)$ with $\rho$ being the state density. It is closely related to the partial-decay width $\Gamma_{v}\left(E_{n}^{*}, J_{n}\right)$ (cf. Sections 4 and 5) by

$$
\Gamma_{v}\left(E_{n}^{*}, J_{n}\right)=\int_{V_{c}}^{E_{n}^{*}-S_{v}} \mathrm{~d} \epsilon_{v} \gamma\left(E_{n}^{*} \rightarrow E_{n+1}^{*}, J_{n}\right),
$$

where $\epsilon_{v}$ is the kinetic energy of the emitted particle labeled by $v$ and $S_{v}$ the corresponding particle-separation energy. $V_{c}$ denotes the Coulomb barrier between the daughter nucleus and the emitted particle. The spectrum of the daughter nucleus $S_{n+1}$ is thus increased by $\delta S_{n+1}$ and should again be normalized to its population, namely

$$
P_{n+1}=\sum_{j^{\prime}}^{N} \sum_{k^{\prime}}^{M} S_{n+1}\left(j^{\prime}, k^{\prime}\right) .
$$

Fig. 1 illustrates a typical two-dimensional spectrum of the daughter nucleus. At the beginning of the decay chain, the spectrum of the mother nucleus has been normalized and is then depleted through the nuclear disintegration towards the daughter nucleus, whose population spectrum is again served as an input for the next step of the cascade, and so forth. In practice, the spectrum of each residual nucleus can be computed by means of the spectral discretization method. It consists in dividing the whole spectrum into a number of energy bins of 0.1 $\mathrm{MeV}$, which can be adjusted by the user, as well as a number of angular-momentum bins, which is limited by the maximum spin of the compound nucleus.

\subsubsection{Time resolution}

The method described above can be improved to take into account the time evolution. In this case, at a time $t_{i}$, the normalization condition (cf. Eq. (11)) becomes

$$
P_{n}\left(t_{i}\right)=\sum_{j^{\prime}}^{N} \sum_{k^{\prime}}^{M} S_{n}\left(t_{i}, j^{\prime}, k^{\prime}\right) .
$$


During a time interval of $\delta t_{i}$, because of the disintegration of the mother nuclei, the population of its daughter $P_{n+1}$ continuously rises. At a given excitation energy $E_{n}^{*}$, this feeding term is simply expressed as $P_{n}\left(t_{i}\right) N_{n}^{i}$ with

$$
N_{n}^{i}=\frac{\Gamma_{v}}{\Gamma_{\text {tot }}^{n}}\left[1-\exp \left(-\Gamma_{\text {tot }}^{n} \delta t_{i}\right)\right]
$$

At the same time, this population exponentially declines, namely $P_{n+1}\left(t_{i}+\delta t_{i}\right)=P_{n+1}\left(t_{i}\right) D_{n+1}^{i}$ with $D_{n+1}^{i}=e^{-\Gamma_{\text {tot }}^{n+1} \delta t_{i}}$. Finally, the iterative equation for the population $P_{n+1}$ reads

$$
P_{n+1}\left(t_{i}+\delta t_{i}\right)=P_{n+1}\left(t_{i}\right) D_{n+1}^{i}+P_{n}\left(t_{i}\right) N_{n}^{i}
$$

When the population spectrum is taken into account, the time dependence is obtained by means of Eq. (12), which leads to

$$
\begin{aligned}
\delta S_{n+1}\left(t_{i}+\delta t_{i}, j, k\right) & =\sum_{J_{n}} \int_{0}^{+\infty} \mathrm{d} E_{n}^{*} S_{n}\left(t_{i}, E_{n}^{*}, J_{n}\right) \\
& \times \frac{\gamma\left(E_{n}^{*} \rightarrow E_{n+1}^{*}, J_{n}\right)}{\Gamma_{\text {tot }}^{n}} \\
& \times\left[1-\exp \left(-\Gamma_{\text {tot }}^{n} \delta t_{i}\right)\right] .
\end{aligned}
$$

In principle, the approach described above can be readily generalized to the multi-channel scheme, but the corresponding representation would get much more complicated. Anyhow, this issue can be handled within the same framework. One can calculate the number of nuclei undergoing fission at each step by looking at the quantity $P_{n}\left(t_{i}\right) F_{n}^{i}$ with $F_{n}^{i}=\Gamma_{f}^{n} / \Gamma_{\text {tot }}^{n}\left(1-e^{-\Gamma_{\text {tot }}^{n} \delta t_{i}}\right)$ for each nucleus $n$ at time $t_{i}$. The average fission time or other dynamical observables can be similarly deduced from a discretized version of Eq. (9). On the basis of this approach, one would be capable of solving more sophisticated dynamics including $\gamma$-ray emission or a transient time during which the fission rate is considerably reduced.

It should be mentioned that the calculation of dynamical variables is often quite time-consuming, owing to the fact that the dynamical decay is a relatively slow process that usually spans several orders of magnitude on the time scale. To overcome this issue, we have introduced an increasing time step, namely $\delta t_{i+1}=\theta \delta t_{i}$. The parameter $\theta$ is employed to enlarge the time step along the cascade, and thus the whole process evolves at very different time scales. The optimum value for the parameter $\theta$ was tested and has been set equal to 1.1 by default, which might lead to a relative error of about $10 \%$ (result tested for single-neutron evaporation). The initial value $\delta t_{0}$ was fixed at $0.01 \hbar / \mathrm{MeV}$.

\section{Heavy-ion fusion}

From this section, we shall concentrate on the physical ingredients included in the KEWPIE2 code. First of all, we make a brief survey for the following fusion process:

$$
\underbrace{a+A}_{\alpha^{\prime}} \longrightarrow C^{*}
$$

Here $\alpha^{\prime}$ refers to a given entrance channel. It should be recalled that only the capture phase has been taken into account in the code.

\subsection{Capture step}

\subsubsection{Semi-classical approximation}

According to some previous studies [63-65], it has been well established that the fusion process can be reasonably described using the coupled-channels approach, especially at sub-barrier energies, as the collective properties of colliding nuclei are taken into consideration. However, owing to its complexity, it would be useful to provide an approximation for estimating fusion cross-sections. To this end, the Wentzel-KramersBrillouin (WKB) approximation is employed to estimate the capture probability, which is simply given by

$$
P_{\text {cap }}\left(E_{\mathrm{cm}}, J_{C}\right)=\exp (-2 \Omega)
$$

with

$$
\Omega=\int_{r_{\text {in }}}^{r_{\text {out }}} \mathrm{d} r \sqrt{\frac{2 \mu_{\alpha^{\prime}}}{\hbar^{2}}\left[V(r)-E_{\mathrm{cm}}\right]},
$$

where $r_{\text {in }}$ and $r_{\text {out }}$ stand for the inner and outer classical turning points, respectively. They satisfy the following relation: $V\left(r_{\text {in }}\right)=V\left(r_{\text {out }}\right)=E_{\mathrm{cm}}$. The reduced mass of the entrance channel is simply given by $\mu_{\alpha^{\prime}}=m_{a} m_{A} /\left(m_{a}+m_{A}\right)$. It should be noted that the above expression would be only appropriate at energies below the Coulomb barrier. In Ref. [66], Kemble proposed a more general solution, namely

$$
P_{\text {cap }}\left(E_{\mathrm{cm}}, J_{C}\right)=\frac{1}{1+\exp (2 \Omega)},
$$

which would be valid at both sub- and above-barrier energies. This generalized WKB formula has been incorporated into the code.

In Eq. (20), $V(r)$ stands for the total potential energy, which can be expressed as follows:

$$
V(r)=V_{N}(r)+V_{\text {coul }}(r)+V_{\text {cent }}(r),
$$

with

$$
V_{\text {coul }}(r)=\frac{Z_{a} Z_{A} e^{2}}{r} \text { and } V_{\text {cent }}(r)=\frac{J_{C}\left(J_{C}+1\right) \hbar^{2}}{2 \mu_{\alpha^{\prime}} r^{2}} .
$$

Regarding the nuclear part $V_{N}(r)$, we adopt the well-known proximity potential [67], whose general form is written as

$$
V_{N}(r)=4 \pi \gamma b \bar{R} \Phi(\xi)
$$

The latest best-fit values of the parameters involved in this formula can be found in Ref. [45], where colliding nuclei have been assumed to be spherical and a Fermi function was employed to describe the density distribution of a nucleus within the droplet model (leptodermous approximation).

It should be noted that, according to some recent studies based upon the couple-channels method, such a simple onedimensional WKB approach would not be suitable for studying heavy-ion fusion at sub-barrier energies, since the coupling between low-lying collective (rotational or vibrational) states in colliding nuclei [65] is not taken into consideration. Some numerical tests clearly show that, using the potential parameterization proposed in Ref. [45], the estimated capture crosssections are in nice agreement with experimental results only 
at energies well above the Coulomb barrier, whereas a significant underestimation has been observed at sub-barrier energies. In fact, this is entirely compatible with most of the couplechannels calculations.

To overcome this issue, we decided to keep using the same parameterization as that included in the HIVAP code [43, 68]. The parameters involved in this potential are summarized as follows:

$$
\begin{aligned}
\bar{R} & =R_{a} R_{A} /\left(R_{a}+R_{A}\right), \text { with } R_{i}=1.126 A_{i}^{1 / 3}, \\
\xi & =\left(r-R_{a}-R_{A}\right) / b, \\
\Delta & =\xi-2.54, \\
\phi(\xi) & =\left\{\begin{array}{l}
0.5 \Delta^{2}-0.0852 \Delta^{3}, \text { if } \xi \leq 1.2511, \\
-3.437 \exp (-\xi / 0.75), \text { otherwise, }
\end{array}\right.
\end{aligned}
$$

where the diffuseness parameter $b$ was set equal to $0.79 \mathrm{fm}$. The factor $4 \pi \gamma$ equals $59 \mathrm{MeV}$, which is considerably larger than the typical values estimated from most proximity-potential models. As a result, a stronger nuclear attraction is generated so as to favor the fusion process at sub-barrier energies. Anyhow, more attention should still be drawn to deformed colliding nuclei where a strong coupling of rotational states to the ground state intervenes at low energies.

Together with Eq. (21), the capture probability can be estimated using numerical integration. For this purpose, the Gaussian-Legendre quadrature rule is employed. For more details, the reader is referred to Appendix A.

\subsubsection{Empirical barrier-distribution method}

In Refs. [19, 46], the authors proposed an empirical approach, which is based upon the assumption that fusion barriers are normally distributed around a mean value $B_{0}$. The capture cross-section thus reads

$$
\sigma_{\text {cap }}=\sqrt{\frac{\pi}{2}} \frac{R_{\text {int }}^{2} w}{E_{\mathrm{cm}}}\left\{\sqrt{\pi} X[1+\operatorname{erf}(X)]+\exp \left(-X^{2}\right)\right\},
$$

where

$$
X=\frac{E_{\mathrm{cm}}-B_{0}}{w \sqrt{2}},
$$

and $\operatorname{erf}(x)$ is the Gaussian error function. Here, $B_{0}$ and $w$ denote the mean value of the barrier distribution and its width, respectively. $R_{\text {int }}$ refers to the relative distance between two colliding nuclei at the contact point. Their values were parameterized by analyzing capture excitation functions for about 50 nuclear reaction systems. Regarding the mean barrier height $B_{0}$, it was parameterized using a cubic polynomial, which reads

$$
B_{0}=0.853315 z+0.0011695 z^{2}-0.000001544 z^{3} \mathrm{MeV} \text {, }
$$

where $z=Z_{a} Z_{A} /\left(A_{a}^{1 / 3}+A_{A}^{1 / 3}\right)$ defined with the charge and mass of the projectile and target nuclei. The parameter $R_{\text {int }}$ has been set equal to $1.16\left(A_{a}^{1 / 3}+A_{A}^{1 / 3}\right) \mathrm{fm}$. As for the width parameter $w$, it can be calculated as follows:

$$
w=D B_{0} \sqrt{\delta_{a}^{2}+\delta_{A}^{2}+\delta_{0}^{2}},
$$

where $\delta_{i}=R_{i}^{2} \beta_{2, i}^{2} / 4 \pi$ with the radii $R_{a}$ and $R_{A}$ defined as $1.15 A_{i}^{1 / 3}$ and their quadrupole deformation parameters $\beta_{2}$ taken from [15]. Finally, $D$ and $\delta_{0}$ are estimated to be $D=0.0421$ $\mathrm{fm}^{-1}$ and $\delta_{0}=0.531 \mathrm{fm}$ [21]. In practice, due to the uncertainty remaining in the width parameter, one usually has to tune its value in order to give reasonable estimates compared with experimental measurements. For this reason, an additional option, which allows the user to modify the mean Coulomb barrier as well as the width parameter value, has been added to the input file.

To take into account different partial waves, one can employ a generalized capture probability for given angular momentum $J_{C}$, which reads

$$
P_{\text {cap }}\left(E_{\mathrm{cm}}, J_{C}\right) \simeq \frac{1}{2}\left[1+\operatorname{erf}\left(\frac{E_{\mathrm{cm}}-B_{\text {eff }}}{w \sqrt{2}}\right)\right],
$$

where the effective barrier $B_{\text {eff }}$ is simply given by

$$
B_{\mathrm{eff}}=B_{0}+\frac{J_{C}\left(J_{C}+1\right) \hbar^{2}}{2 \mu_{\alpha^{\prime}} R_{\mathrm{int}}^{2}}
$$

\subsection{Formation step}

As previously mentioned, the fusion hindrance is not dealt with in the current version of the code due to the fact that some theoretical ambiguities still persist. In the case of hindered reactions, it would be necessary to account for the dynamical evolution from a di-nuclear system towards a mono-nuclear configuration. Unfortunately, no canonical description of such a process has been generally accepted so far.

In most cases, the formation probability entering Eq. (3) should be separately estimated, for instance, by using a multi-dimensional Langevin equation [58, 59]. In the onedimensional case, a simple analytical formula for the diffusion probability of surmounting the conditional saddle point can be obtained using an inverted-parabolic potential barrier $[53,54,69,70]$. More recently, by making use of a simplified model [71], we proposed that the rapid evolution of the "neck" variable might lead to a large initial shift along the radial elongation, which provides a primary justification for the injection point phenomenologically introduced in Refs. [19, 72]. To eliminate the existing ambiguities, further consideration will be required, in order to give a better understanding of the fusion hindrance phenomenon and to make quantitative predictions for guiding future experiments on the synthesis of super-heavy elements.

Based upon the above discussions, it would be helpful to accommodate other theoretical models which somehow take into account the influence of fusion hindrance on the predicted cross-sections. This can be done by reading a user-defined data file that contains the calculated results for all partialwave contributions to the fusion excitation function, namely $\sigma_{\text {cap }}\left(E_{\mathrm{cm}}, J_{C}\right) P_{\text {form }}\left(E_{\mathrm{cm}}, J_{C}\right)$.

\section{Light-particle evaporation and $\gamma$-ray emission}

\subsection{Detailed balance}

We now consider a compound nucleus, $C$, with total excitation energy $E_{C}^{*}$, from which a light particle, $b$, is ejected and 
a residual nucleus, $B$, thus survives. This process can be illustrated as follows:

$$
C^{*} \longrightarrow \underbrace{B+b}_{\beta^{\prime}},
$$

where $\beta^{\prime}$ denotes a specified exit channel and the whole process is assumed to take place within a finite region characterized by a certain volume of $\mathcal{V}$. The total kinetic energy of the exit channel in the centre-of-mass frame is simply given by $\epsilon_{\beta^{\prime}}=\mu_{\beta^{\prime}} v_{b}^{2} / 2$, where $v_{b}$ represents the velocity of $b$ relative to $B$. The reduced mass of the binary system is denoted by $\mu_{\beta^{\prime}}$, namely $\mu_{\beta^{\prime}}=m_{b} m_{B} /\left(m_{b}+m_{B}\right)$.

According to the reciprocity theorem [16], the decay rate $R_{C^{*} \rightarrow B+b}$ is related to its time-reversed decay rate $R_{b+B \rightarrow C^{*}}$ by

$$
R_{C^{*} \rightarrow B+b}=R_{b+B \rightarrow C^{*}} \frac{\rho_{\beta^{\prime}}\left(E_{\beta^{\prime}}\right)}{\rho_{C}\left(E_{C}^{*}\right)},
$$

where $E_{\beta^{\prime}}$ represents the total energy of the binary system in the centre-of-mass frame, which is simply given by $E_{\beta^{\prime}}=\epsilon_{\beta^{\prime}}+E_{B}^{*}=$ $E_{C}^{*}-S_{b}$ with $S_{b}$ being the separation energy of the emitted particle. $\rho_{\beta^{\prime}}\left(E_{\beta^{\prime}}\right)$ corresponds to the total state density of the binary system obtained by convolution. Thus, one obtains the following expression for the decay width:

$$
\frac{\Gamma_{b}}{\hbar}=\frac{\rho_{\beta^{\prime}}\left(E_{\beta^{\prime}}\right)}{\rho_{C}\left(E_{C}^{*}\right)} R_{b+B \rightarrow C^{*}} .
$$

More explicitly, one has

$$
\frac{\Gamma_{b}}{\hbar}=\frac{\rho_{\beta^{\prime}}\left(E_{\beta^{\prime}}\right)}{\rho_{C}\left(E_{C}^{*}\right)} \frac{v_{b}}{\mathcal{V}} \sigma_{\text {inv }}^{b}
$$

where $\sigma_{\text {inv }}^{b}$ denotes the cross-section of the time-reversed reaction $b+B \longrightarrow C^{*}$. It should be borne in mind that the spin degeneracy of the emitted particle, namely $2 s_{b}+1$, has been taken into account in the combined state density $\rho_{\beta^{\prime}}\left(E_{\beta^{\prime}}\right)$, whereas the spins of compound and residual nuclei shall further be considered within the Hauser-Feshbach formalism.

\subsection{Weisskopf-Ewing model}

With the help of Eq. (34), one obtains the following wellknown formula [16, 17, 73]:

$$
\Gamma_{b}\left(E_{C}^{*}\right)=\frac{\left(2 s_{b}+1\right) \mu_{\beta^{\prime}}}{\pi^{2} \hbar^{2}} \int_{V_{c}}^{\epsilon_{b}^{\max }} \frac{\sigma_{\mathrm{inv}}^{b}\left(\epsilon_{\beta^{\prime}}\right) \rho_{B}\left(E_{B}^{*}\right)}{\rho_{C}\left(E_{C}^{*}\right)} \epsilon_{\beta^{\prime}} \mathrm{d} \epsilon_{\beta^{\prime}},
$$

where $V_{c}$ denotes the Coulomb barrier between the residual nucleus and the emitted particle, which is equal to zero for neutrons. The upper bound for the integral $\epsilon_{\beta^{\prime}}^{\max }$ is taken to be $E_{C}^{*}-S_{b}$. In the case of neutrons, the cross section for the timereversed reaction is given by [74]

$$
\sigma_{\text {inv }}^{b}\left(\epsilon_{\beta^{\prime}}\right)=g_{0}\left(1+\frac{g_{1}}{\epsilon_{\beta^{\prime}}}\right) \pi \tilde{R}^{2}
$$

where $g_{0}=0.76+1.93 A_{B}^{-1 / 3}, g_{0} g_{1}=1.66 A_{B}^{-2 / 3}-0.05$ and $\tilde{R}=1.7 A_{B}^{1 / 3} \mathrm{fm}$. For charged particles, one has [75]

$$
\sigma_{\text {inv }}^{b}\left(\epsilon_{\beta^{\prime}}\right)=\left(1-\frac{V_{c}}{\epsilon_{\beta^{\prime}}}\right) \pi \tilde{R}^{2},
$$

with $\tilde{R}=r_{0} A_{B}^{1 / 3}+R_{b}, r_{0}=1.42 \mathrm{fm}$ and $V_{c}=Z_{b} Z_{B} e^{2} /\left(r_{e} A_{B}^{1 / 3}+\right.$ $R_{b}$ ). For protons, $R_{b}=1.44 \mathrm{fm}$ and $r_{e}=1.81 \mathrm{fm}$. For $\alpha$ particles, $R_{b}=2.53 \mathrm{fm}$ and $r_{e}=2.452-0.408 \log _{10}\left(Z_{b} Z_{B}\right)$ fm. Here $A_{B}$ and $Z_{B}$ correspond to the mass and charge of the daughter nucleus $B$, respectively.

\subsection{Hauser-Feshbach formalism}

Up to now, we have ignored the spin degrees of freedom for compound and residual nuclei. To incorporate it into the code, one needs to rewrite the reciprocity theorem (cf. Eq. (34)) in the following form:

$$
\frac{\Gamma_{b}}{\hbar}=\frac{\left(2 s_{B}+1\right) \rho_{B+b}\left(E_{\beta^{\prime}}, s_{B}\right)}{\left(2 J_{C}+1\right) \rho_{C}\left(E_{C}^{*}, J_{C}\right)} \frac{v_{b}}{V} \sigma_{\text {inv }}^{b} .
$$

Moreover, within the framework of the Hauser-Feshbach formalism [18], $\sigma_{\text {inv }}^{b}$ can be evaluated as follows:

$$
\sigma_{\mathrm{inv}}^{b}=\frac{\pi}{k^{2}} \frac{2 J_{C}+1}{\left(2 s_{b}+1\right)\left(2 s_{B}+1\right)} \sum_{s_{\beta^{\prime}}=\left|s_{B}-s_{b}\right|}^{s_{B}+s_{b}} \sum_{\beta_{\beta^{\prime}}=\left|J_{C}-s_{\beta^{\prime}}\right|}^{J_{C}+s_{\beta^{\prime}}} T_{l_{\beta^{\prime}}}\left(\epsilon_{b}\right),
$$

where the channel spin is denoted by $\overrightarrow{s_{\beta^{\prime}}}=\overrightarrow{s_{B}}+\overrightarrow{s_{b}}$ and the total orbital angular momentum in the centre-of-mass frame by $\overrightarrow{l_{\beta^{\prime}}}$. Substituting Eq. (39) into Eq. (38) and integrating over all possible kinetic energies, a generalized expression is obtained for the partial decay width, which reads

$$
\begin{aligned}
\Gamma_{b}\left(E_{C}^{*}, J_{C} ; s_{B}\right)=\int_{0}^{\epsilon_{\beta^{\prime}}^{\max }} & \sum_{s_{\beta^{\prime}}=\left|s_{B}-s_{b}\right|}^{s_{\beta^{\prime}}+s_{b}} \sum_{\beta_{\beta^{\prime}}}^{J_{C}+s_{\beta^{\prime}}-s_{\beta^{\prime}} \mid} \rho_{B}\left(E_{B}^{*}, s_{B}\right) \\
& \times \frac{T_{l_{\beta^{\prime}}}\left(\epsilon_{\beta^{\prime}}\right) \mathrm{d} \epsilon_{\beta^{\prime}}}{2 \pi \rho_{C}\left(E_{C}^{*}, J_{C}\right)} .
\end{aligned}
$$

Finally, after summing over all possible values of the spin $s_{B}$, one obtains

$$
\begin{aligned}
\Gamma_{b}\left(E_{C}^{*}, J_{C}\right)=\int_{0}^{\epsilon_{\beta^{\prime}}^{\max }} \sum_{s_{B}} \sum_{s_{\beta^{\prime}}=\left|s_{B}-s_{b}\right| l_{\beta^{\prime}}=\left|J_{C}-s_{\beta^{\prime}}\right|}^{s_{B}+s_{b}} \rho_{B}\left(E_{B}^{*}, s_{B}\right) \\
\times \frac{T_{l_{\beta^{\prime}}}\left(\epsilon_{\beta^{\prime}}\right) \mathrm{d} \epsilon_{\beta^{\prime}}}{2 \pi \rho_{C}\left(E_{C}^{*}, J_{C}\right)} .
\end{aligned}
$$

In obtaining Eq. (41), one first couples the spin vectors $\overrightarrow{s_{B}}$ and $\overrightarrow{s_{b}}$ to form $\overrightarrow{s_{\beta^{\prime}}}$, which is followed by coupling of $\overrightarrow{l_{\beta^{\prime}}}$ and $\overrightarrow{s_{\beta^{\prime}}}$ to generate the total angular momentum $\overrightarrow{J_{C}}$. This is just a matter of choice. In the KEWPIE2 code, we adopt another procedure, that is, coupling of $\overrightarrow{s_{b}}$ and $\overrightarrow{l_{\beta^{\prime}}}$ to form $\overrightarrow{j_{b}}$, and then combining with $\overrightarrow{s_{B}}$ to generate the spin vector of the compound nucleus $\overrightarrow{J_{C}}$. In this case, one obtains a similar formula for the particleevaporation width, namely

$$
\begin{aligned}
\Gamma_{b}\left(E_{C}^{*}, J_{C}\right)=\int_{0}^{\epsilon_{\beta^{\prime}}^{\max }} \sum_{l_{\beta^{\prime}=0}}^{l_{\beta^{\prime}}^{\max }} \sum_{j_{b}=\left|l_{\beta^{\prime}}-s_{b}\right|}^{l_{\beta^{\prime}}+s_{b}} \sum_{s_{B}=\left|J_{C}-j_{b}\right|}^{J_{C}+j_{b}} \rho_{B}\left(E_{B}^{*}, s_{B}\right) \\
\times \frac{T_{l_{\beta^{\prime}}, j_{b}}\left(\epsilon_{\beta^{\prime}}\right) \mathrm{d} \epsilon_{\beta^{\prime}}}{2 \pi \rho_{C}\left(E_{C}^{*}, J_{C}\right)} .
\end{aligned}
$$


Note that the summation over $l_{\beta^{\prime}}$ terminates as long as the transmission coefficient vanishes, namely at the upper limit $l_{\beta^{\prime}}^{\max }$. Such a scheme, which has been incorporated into the current version of the code, would be more suitable when the spinorbit interaction is considered in the nuclear optical potential. In this case, as seen in Eq. (42), the transmission coefficient $T_{l_{\beta^{\prime}}, j_{b}}$ should be dependent on both $l_{\beta^{\prime}}$ and $j_{b}$.

\subsubsection{Optical potential}

In the previous version of the code, the BecchettiGreenless [76] and Wilmore-Hodgson [77] empirical opticalpotential parameterizations were introduced for incident neutrons and protons, respectively. However, they were both developed during the 1960s and since then, there have been many important advances in our understanding of the nuclear optical potential. Furthermore, more accurate data for scattering crosssections have become available and the computational ability to optimize models parameters has been largely improved. Based upon these considerations, a recent optical parameterization proposed by Varner et al. [78] has been included in the current version of the code for both protons and neutrons. In this model, the local optical potential for nucleon-nucleus scattering is expressed as

$$
\begin{aligned}
V_{\mathrm{opt}}(r) & =-V_{r} f_{\mathrm{ws}}\left(r, R_{0}, a_{0}\right)-i W_{v} f_{\mathrm{ws}}\left(r, R_{w}, a_{w}\right) \\
& -i W_{s}\left(-4 a_{w}\right) \frac{\mathrm{d}}{\mathrm{d} r} f_{\mathrm{ws}}\left(r, R_{w}, a_{w}\right) \\
& +\frac{\lambda_{\pi}^{2 \vec{\sigma} \cdot \overrightarrow{l_{\beta^{\prime}}}}}{r}\left(V_{\mathrm{so}}+i W_{\mathrm{so}}\right) \frac{\mathrm{d}}{\mathrm{d} r} f_{\mathrm{ws}}\left(r, R_{\mathrm{so}}, a_{\mathrm{so}}\right),
\end{aligned}
$$

with $\lambda_{\pi}^{2}=2 \mathrm{fm}^{2}$ and

$$
f_{\mathrm{ws}}(r, R, a)=\frac{1}{1+\exp \left(\frac{r-R}{a}\right)} .
$$

The Coulomb potential for the emission of protons is simply given by

$$
V_{c}=\left\{\begin{array}{cc}
\frac{Z_{B} e^{2}}{r}, & \text { for } r \geq R_{c}, \\
\frac{Z_{B} e^{2}}{2 R_{c}}\left(3-\frac{r^{2}}{R_{c}^{2}}\right), & \text { for } 0 \leq r \leq R_{c},
\end{array}\right.
$$

where $R_{c}$ represents the Coulomb radius. As for the parameters involved within this parameterization, the reader is referred to [78]. In addition, another more recent optical potential parameterization [79], which is also valid for both neutrons and protons with incident energies from $1 \mathrm{keV}$ up to $200 \mathrm{MeV}$, has also been included in the code.

Then, let us take a look at $\alpha$ particles, whose optical potential is taken to be [80]

$$
\begin{aligned}
V_{\mathrm{opt}}(r) & =-\left[\left(c_{0}+c_{1} \frac{Z_{B}}{A_{B}^{1 / 3}}+c_{2} E_{\alpha}\right) f_{\mathrm{ws}}\left(r, r_{v}, a_{v}\right)\right] \\
& -i\left[\left(c_{0}^{\prime}+c_{1}^{\prime} A_{B}^{1 / 3}+c_{2}^{\prime} E_{\alpha}\right) f_{\mathrm{ws}}\left(r, r_{w}, a_{w}\right)\right],
\end{aligned}
$$

and the diffuseness parameters are given as follows:

$$
\begin{aligned}
& a_{v}=d_{0}+d_{1} A_{B}^{1 / 3}, \\
& a_{w}=d_{0}^{\prime}+d_{1}^{\prime} A_{B}^{1 / 3} .
\end{aligned}
$$

The best-fit values of the above parameters, which were obtained by optimizing the $\chi^{2}$ function for the scattering data at energies above $80 \mathrm{MeV}$, can be found in Ref. [80]. Note that this parameterization has been extended to lower energies by Avrigeanu et al. [81].

\subsubsection{Transmission coefficient}

The usual procedure to calculate the transmission coefficient $T_{l_{\beta^{\prime}}, j_{b}}\left(\epsilon_{\beta^{\prime}}\right)$ consists in solving numerically the Schrödinger equation, which reads

$$
\left\{\frac{\mathrm{d}^{2}}{\mathrm{~d} r^{2}}-\frac{l_{\beta^{\prime}}\left(l_{\beta^{\prime}}+1\right)}{r^{2}}-\frac{2 \mu_{\beta^{\prime}}}{\hbar^{2}}\left[V_{c}(r)+V_{\mathrm{opt}}(r)-E_{\mathrm{cm}}\right]\right\} u(r)=0,
$$

from $r=0$ to infinity by means of the modified Numerov method. The computation time is mainly dependent on the stepsize $\Delta r$ for the numerical integration and the maximal distance $R_{\max }$ that is much larger than the range of the nuclear interaction so as to match the solutions of Eq. (48) to the Coulomb functions. Their default values have been chosen to be $0.05 \mathrm{fm}$ and $50 \mathrm{fm}$, respectively.

Special attention should be drawn to the case of nucleons, both of which have a spin $s_{b}=1 / 2$. Hence, for each $l_{\beta^{\prime}}$, one has two eigenstates for $j_{b}$, namely $l_{\beta^{\prime}}-1 / 2$ and $l_{\beta^{\prime}}+1 / 2$, as a result of the spin-orbit coupling. This has actually been considered in Eq. (43), where the coupling term $\vec{\sigma} \cdot \overrightarrow{l_{\beta^{\prime}}}$ can be rewritten in terms of $\overrightarrow{j_{b}}$ and $\overrightarrow{\beta_{\beta^{\prime}}}$ as follows:

$$
\vec{\sigma} \cdot \overrightarrow{l_{\beta^{\prime}}}=j_{b}\left(j_{b}+1\right)-l_{\beta^{\prime}}\left(1+l_{\beta^{\prime}}\right)-3 / 4
$$

where $\vec{\sigma}$ denotes the Pauli matrix. By making use of this definition, the transmission coefficient $T_{l_{\beta^{\prime}}, j_{b}}$ present in Eq. (42) is automatically computed at each iteration.

Finally, as pointed out by Alexander et al. [82], the transmission coefficient obtained from the optical model would not be appropriate for treating the evaporation of light particles. Indeed, statistical-model calculations are only concerned with the evaporation of a particle from an excited nucleus or its timereversed process, namely absorption, whereas usual opticalpotential models also comprise other effects related to inelastic scattering, size resonances, etc. As a consequence, the transmission coefficient might not approach unity at energies well above the barrier (transparency effect). To ensure a full absorption within the potential well (elimination of other effects), as discussed in Ref. [82], one only needs to keep the real part of the optical potential and the incoming-wave boundary condition (IWBC) [83] is thus employed to estimate the transmission coefficient $T_{l_{\beta^{\prime}}, j_{b}}$. In our case, the IWBC would be crucial for both neutrons and protons, but appears slightly less important for $\alpha$ particles because they already experience strong absorption inside the Coulomb barrier. 


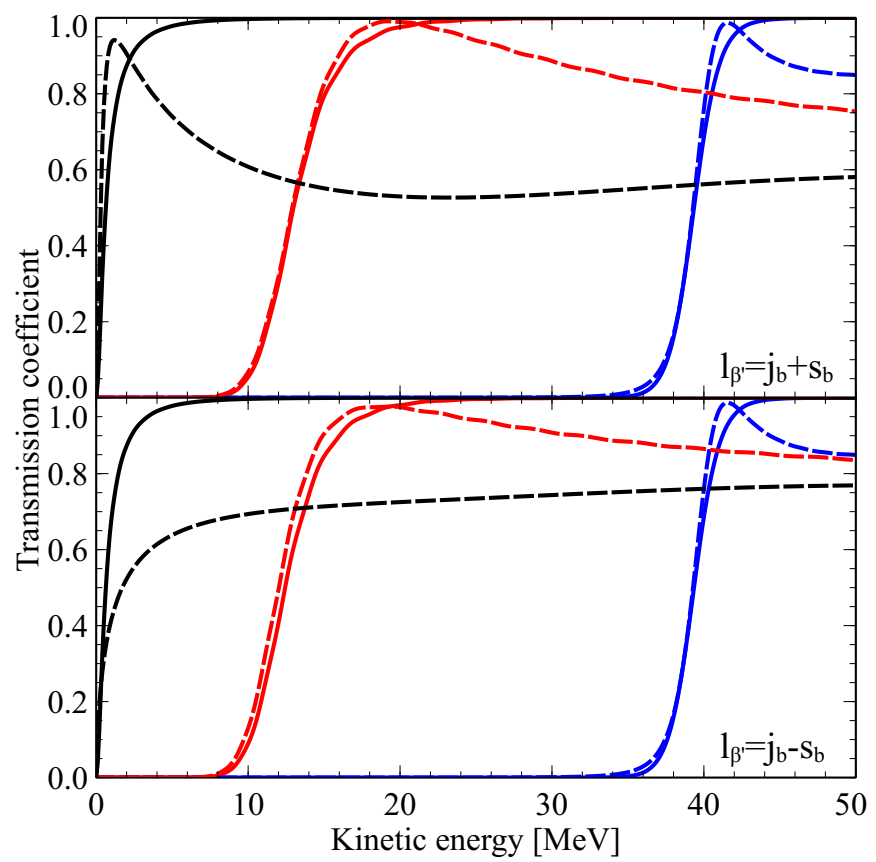

Fig. 2. Comparison of the calculated transmission coefficients based upon the optical and IWBC models as a function of the kinetic energy of the emitted particle in the centre-of-mass frame. The solid and dashed curves (black for neutrons, red for protons and blue for $\alpha$ particles) correspond to the calculations performed with the IWBC and optical models, respectively. Here, the mother nucleus is ${ }^{243} \mathrm{Cm}$ and $j_{b}=1$. Two spin-orbit components have been shown ( $s_{b}=1 / 2$ for neutrons and protons, $s_{b}=0$ for $\alpha$ particles.).

Fig. 2 shows a comparison between the transmission coefficients calculated within the optical and IWBC models. It can be readily observed that, under the IWBC, the transmission coefficient gradually tends to unity, whereas this is not the case for the optical model. Regarding the numerical implementation of the IWBC, please refer to Appendix B for more details.

\section{4. $\gamma$-ray emission}

Apart from the evaporation of light particles, the residual nucleus can also emit $\gamma$ rays. Basically, the $\gamma$-ray emission would become dominant when the excitation energy is lower than both the fission and particle-evaporation thresholds. In the case of very-heavy and super-heavy nuclei, since the fission barrier could be much smaller than the particle-evaporation threshold, the competition between $\gamma$-ray emission and fission would be essential at low excitation energies. Moreover, some recent studies $[10,84]$ showed that the distribution of spin and excitation energy of the residual nucleus, which results from the $\gamma$-ray emission, can be employed to explore the fission barrier in heavy nuclei.

By analogy with the Hauser-Feshbach formalism, the $\gamma$ decay width is defined as

$$
\begin{aligned}
\Gamma_{\gamma}\left(E_{C}^{*}, J_{C}\right)=\int_{0}^{E_{C}^{*}} \sum_{\lambda} \sum_{s_{B}=\left|J_{C}-\lambda\right|}^{J_{C}+\lambda} \rho_{B}\left(E_{C}^{*}-\epsilon_{\gamma}, s_{B}\right) \\
\times \frac{T_{X \lambda}\left(\epsilon_{\gamma}\right) \mathrm{d} \epsilon_{\gamma}}{2 \pi \rho_{C}\left(E_{C}^{*}, J_{C}\right)}
\end{aligned}
$$

where $T_{X \lambda}$ stands for the transmission coefficient for the $\gamma$ decay of type $X$ (electric or magnetic transition), which reads

$$
T_{X \lambda}\left(\epsilon_{\gamma}\right)=2 \pi \epsilon_{\gamma}^{2 \lambda+1} f_{X \lambda}\left(\epsilon_{\gamma}\right)
$$

with $\lambda$ being the multi-polarity and $f_{X \lambda}\left(\epsilon_{\gamma}\right)$ the radiative strength function.

Traditional methods of estimating radiative strength functions are usually based upon the Weisskopf single-particle model [85]. Nevertheless, the nuclear-structure effects are known to modify the Weisskopf estimates by introducing an enhancement factor up to two or three orders of magnitude. Fortunately, one can make great improvements on this issue with the help of the Brink-Axel hypothesis [86, 87], which states that, at energies around the resonance, the radiative strength function is assumed to have a Lorentzian-like line shape. Within this framework, the radiative strength function is generally expressed as

$$
f_{X \lambda}^{\mathrm{SLO}}\left(\epsilon_{\gamma}\right)=\frac{26 \times 10^{-8}}{2 \lambda+1} \times \sigma_{r} \Gamma_{r} \epsilon_{\gamma}^{3-2 \lambda} \frac{\Gamma_{r}}{\left(\epsilon_{\gamma}^{2}-E_{r}^{2}\right)^{2}+\epsilon_{\gamma}^{2} \Gamma_{r}^{2}},
$$

where $\sigma_{r}$ and $E_{r}$ respectively correspond to the cross-section and energy of giant-dipole resonance, and the resonance width $\Gamma_{r}$ is considered to be constant. The unit of the strength function is $\mathrm{MeV}^{-3}$. Eq. (52) is usually called the Standard Lorentzian model (SLO) and has largely been employed for fitting to experimental data.

The $\gamma$-ray emission would play a crucial role in the calculation of fission times. Accordingly, some recent parameterizations based upon the SLO for calculating the radiative strength function have been taken into consideration in our model and will be briefly introduced in this subsection.

\subsubsection{E1 strength function}

It is well known that the $\gamma$-decay strength function is mainly governed by the giant dipole resonance. An improved version of the SLO, known as the Enhanced Generalized Lorentzian model (EGLO), was proposed in Ref. [88] for the calculation of the E1 strength function,

$$
\begin{aligned}
f_{E 1}^{\mathrm{EGLO}}\left(\epsilon_{\gamma}\right) & =8.674 \cdot 10^{-8} \times \sigma_{r} \Gamma_{r} \\
& \times\left[\frac{\epsilon_{\gamma} \Gamma_{K}\left(\epsilon_{\gamma}, T_{f}\right)}{\left(\epsilon_{\gamma}^{2}-E_{r}^{2}\right)^{2}+\epsilon_{\gamma}^{2} \Gamma_{K}^{2}\left(\epsilon_{\gamma}, T_{f}\right)}+\frac{\left.0.7 \Gamma_{K}\left(\epsilon_{\gamma}, T_{f}\right)\right|_{\epsilon_{\gamma}=0}}{E_{r}^{3}}\right],
\end{aligned}
$$

where the energy-dependent collisional width $\Gamma_{K}\left(\epsilon_{\gamma}, T_{f}\right)$ is given by

$$
\Gamma_{K}\left(\epsilon_{\gamma}, T_{f}\right)=\chi\left(\epsilon_{\gamma}\right) \frac{\Gamma_{r}}{E_{r}^{2}}\left[\epsilon_{\gamma}^{2}+\left(2 \pi T_{f}\right)^{2}\right],
$$

which is assumed to be proportional to an empirical function $\chi_{\gamma}\left(\epsilon_{\gamma}\right)$ that is defined as

$$
\chi\left(\epsilon_{\gamma}\right)=\tau+(1-\tau) \frac{\epsilon_{\gamma}-\epsilon_{0}}{E_{r}-\epsilon_{0}},
$$

where the factor $\tau$ is dependent on the model employed to describe the nuclear state density, whereas $\epsilon_{0}$ is kept fixed at 
4.5 MeV. In our model, we adopt the following parameterization [89] for $\tau$ :

$\tau=\left\{\begin{array}{cc}1, & A_{C}<148, \\ 1+0.09\left(A_{C}-148\right)^{2} \exp \left[-0.18\left(A_{C}-148\right)\right], & A_{C} \geq 148 .\end{array}\right.$

Recalling that $A_{C}$ refers to the mass number of the compound nucleus. In the above formulas, $T_{f}$ represents the nuclear temperature of the final state, which is closely related to the excitation energy of the residual nucleus. Within the back-shifted Fermi-gas model, it can be approximately estimated as follows:

$$
T_{f}=\sqrt{\left(E_{C}^{*}-\Delta_{\text {pair }}-\epsilon_{\gamma}\right) / a\left(E_{C}^{*}-\Delta_{\text {pair }}\right)},
$$

where $\Delta_{\text {pair }}$ denotes the paring energy and $a\left(E_{C}^{*}-\Delta_{\text {pair }}\right)$ the energy-dependent level-density parameter (cf. Section 6).

However, the EGLO expression for the $\gamma$-decay strength function contradicts some aspects of microscopic theoretical studies [90-92]. Particularly, it has been established that the shape of the $\gamma$-decay strength function is not consistent with the nuclear linear response theory for heated nuclei. This drawback was approximately avoided in some recent studies [40, 41], where a new closed-form formula that is referred to as the Modified Lorentzian model (MLO) was proposed, namely

$$
\begin{aligned}
f_{E 1}^{\mathrm{MLO}}\left(\epsilon_{\gamma}\right) & =8.674 \cdot 10^{-8} \times \sigma_{r} \Gamma_{r} \Lambda\left(\epsilon_{\gamma}, T_{f}\right) \\
& \times \frac{\epsilon_{\gamma} \Gamma\left(\epsilon_{\gamma}, T_{f}\right)}{\left(\epsilon_{\gamma}^{2}-E_{r}^{2}\right)^{2}+\epsilon_{\gamma}^{2} \Gamma^{2}\left(\epsilon_{\gamma}, T_{f}\right)},
\end{aligned}
$$

where $\Lambda\left(\epsilon_{\gamma}, T_{f}\right)$ refers to the scaling factor that reflects the enhancement of the strength function in the warm nuclei, which is usually defined as

$$
\Lambda\left(\epsilon_{\gamma}, T_{f}\right)=1 /\left[1-\exp \left(-\epsilon_{\gamma} / T_{f}\right)\right]
$$

Regarding the width function $\Gamma\left(\epsilon_{\gamma}, T_{f}\right)$, different semiempirical expressions have been introduced in the MLO approach (MLO1, MLO2, MLO3), but obviously they should be in close agreement. In the KEWPIE2 code, we adopt the simplified version of the MLO model, namely the SMLO, which would be preferable due to its simple numerical implementation. More concretely, the energy-dependent width $\Gamma\left(\epsilon_{\gamma}, T_{f}\right)$ within this model is simply expressed as $\Gamma_{r} E_{C}^{*} / E_{r}$.

In Refs. [93, 94], a new parameterization for $\sigma_{r}, E_{r}$ and $\Gamma_{r}$ appearing in Eqs. (53) and (58) was proposed. It can be summarized as follows:

$$
\begin{aligned}
& E_{r}=a_{1}\left(1+b_{1} I_{C}^{2}\right) A_{C}^{-1 / 3}+a_{2}\left(1+b_{2} I_{C}^{2}\right) A_{C}^{-1 / 6} \mathrm{MeV}, \\
& \Gamma_{r}=a_{3} E_{r}^{\delta} \mathrm{MeV}, \\
& S_{r}=\pi \sigma_{r} \Gamma_{r} / 2=60 a_{4} N_{C} Z_{C} / A_{C} \mathrm{mb} \cdot \mathrm{MeV},
\end{aligned}
$$

where $I_{C}$ corresponds to the relative neutron excess of the compound nucleus, namely $I_{C}=\left(A_{C}-2 Z_{C}\right) / A_{C}$. Note that this might only be valid for spherical nuclei. For axially deformed nuclei, the $E 1$ strength function can be expressed as the sum of two components, namely $\left(E_{r, 1}, \Gamma_{r, 1}, \sigma_{r, 1}\right)$ and $\left(E_{r, 2}, \Gamma_{r, 2}, \sigma_{r, 2}\right)$, which result from the collective motion along and perpendicular to the axis of symmetry, respectively. In practice, one can employ the following parametrization $[89,95]$ :

$$
\begin{aligned}
E_{r, 1} & =E_{r, 2} /\left(0.911 a_{0} / b_{0}+0.089\right), \\
E_{r, 2} & =E_{r}\left[1-1.51 \times 10^{-2}\left(a_{0}^{2}-b_{0}^{2}\right)\right], \\
\Gamma_{r, 1} & =0.026 E_{r, 1}^{1.91}, \Gamma_{r, 2}=0.026 E_{r, 2}^{1.91}, \\
\sigma_{r, 1} & =\sigma_{r} / 3, \sigma_{r, 2}=2 \sigma_{r} / 3,
\end{aligned}
$$

where $E_{r}, \Gamma_{r}$, and $\sigma_{r}$ can be evaluated from Eq. (60). Here $a_{0}$ and $b_{0}$ denote the relative semi-axes of a spheroid that are related to the quadrupole deformation parameter $\beta_{2}$ by $a_{0}=(1+$ $\left.\alpha_{2}\right) / \lambda$ and $b_{0}=\left(1-0.5 \alpha_{2}\right) / \lambda$ with $\beta_{2}=\sqrt{4 \pi / 5} \alpha_{2}$, respectively. $\lambda$ is expressed in terms of $\alpha_{2}$, namely $\lambda^{3}=1+3 \alpha_{2}^{2} / 5+2 \alpha_{2}^{3} / 35$. The quadrupole deformation parameter $\beta_{2}$ is given in Ref. [15].

In the KEWPIE2 code, both the EGLO and SMLO approaches have been taken into account. The parameter values are summarized as follows:

- For EGLO, one has $\delta=1.91, a_{1}=27.469 \pm 0.009, a_{2}=$ $22.063 \pm 0.004, a_{3}=0.02691 \pm 0.00004$ and $a_{4}=1.2224 \pm$ $0.0019 . b_{1}$ and $b_{2}$ are both taken to be 0.0 .

- For SMLO, one has $\delta=1.0, a_{1}=28.69 \pm 0.01, a_{2}=$ $21.731 \pm 0.004, a_{3}=0.33078 \pm 0.00047$ and $a_{4}=1.2669 \pm$ $0.0021 . b_{1}$ and $b_{2}$ are both taken to be 0.0 .

\subsubsection{E2 and M1 strength functions}

As regards other higher-order $\gamma$-ray emissions, such as the E2 and M1 transitions, we adopt the SLO form (cf. Eq. (52)), as recommended in Ref. [89]. The default parameter values are given by

$$
\begin{aligned}
E_{r} & =63 A_{C}^{-1 / 3} \mathrm{MeV}, \\
\Gamma_{r} & =6.11-0.012 A_{C} \mathrm{MeV}, \\
\sigma_{r} & =1.5 \times 10^{-4} \frac{Z_{C}^{2} E_{r}^{2}}{A_{C}^{1 / 3} \Gamma_{r}} \mathrm{mb}
\end{aligned}
$$

for $E 2$ emission and by

$$
\begin{aligned}
E_{r} & =41 A_{C}^{-1 / 3} \mathrm{MeV}, \\
\Gamma_{r} & =4.0 \mathrm{MeV}, \\
\sigma_{r} & =1.0 \mathrm{mb}
\end{aligned}
$$

for $M 1$ emission, respectively.

It should be mentioned that higher-order emissions are generally much weaker than the giant-dipole one, which would be dominant in $\gamma$-decay width calculations. Typically, based upon the Weisskopf single-particle model, one has the following crude estimates of their orders of magnitude [30]:

$$
\frac{f_{E(\lambda+1)}}{f_{E(\lambda)}} \simeq 3.7 \cdot 10^{-5} A_{C}^{2 / 3} \epsilon_{\gamma}^{2}\left[\frac{3+\lambda}{5+\lambda}\right]^{2}
$$

and

$$
\frac{f_{M(\lambda+1)}}{f_{E(\lambda+1)}} \simeq 0.307 A_{C}^{-2 / 3}
$$




\section{Nuclear fission}

Nuclear fission is the ruling decay channel of heavy and super-heavy nuclei. It basically competes with the emission of light particles and thus determines the survival probability of the residual nucleus. In the case of super-heavy nuclei, the fission-barrier height is usually lower than the particle-emission threshold. However, compared with the evaporation process, nuclear fission is considered much more complicated to describe since large-amplitude collective deformations come into play.

In the KEWPIE2 code, the fission-decay width is estimated within the standard Bohr-Wheeler transition-state model [32]:

$$
\Gamma_{f}^{\mathrm{BW}}\left(E_{C}^{*}, J_{C}\right)=\frac{1}{2 \pi \rho_{C}^{\mathrm{gs}}\left(E_{C}^{*}, J_{C}\right)} \int_{0}^{E_{\mathrm{gs}}^{*}-B_{f}} \rho_{C}^{\mathrm{sd}}\left(E_{\mathrm{sd}}^{*}, J_{C}\right) \mathrm{d} \epsilon_{f},
$$

where the excitation energy at the saddle point $E_{\mathrm{sd}}^{*}$ is equal to $E_{\mathrm{C}}^{*}-B_{f}-\epsilon_{f}$. One could also introduce a penetration factor, namely $T_{\text {fiss }}\left(\epsilon_{f}\right)$, which corresponds to the well-known HillWheeler transmission coefficient [96]. It takes the following form:

$$
T_{\text {fiss }}\left(\epsilon_{f}\right)=\frac{1}{1+\exp \left(-\frac{2 \pi \epsilon_{f}}{\hbar \omega_{\text {sd }}}\right)}
$$

where $\hbar \omega_{\text {sd }}$ represents the potential curvature at the saddle point. The default value of $\hbar \omega_{\text {sd }}$ has been fixed at $1.0 \mathrm{MeV}$, which can be adjusted by the user. By default, Eq. (66) is employed without taking the penetration factor.

From a dynamical point of view, the fission-decay width evaluated by Eq. (66) can be refined by introducing a correction factor [33], which reads

$$
K=\sqrt{1+\left(\frac{\beta}{2 \omega_{\mathrm{sd}}}\right)^{2}}-\frac{\beta}{2 \omega_{\mathrm{sd}}},
$$

which takes into account the effect of viscosity on the fission process. Here $\beta$ stands for the reduced friction parameter whose default value has been set to $5.0 \mathrm{zs}^{-1}$, which can be tuned by means of the input file. On the other hand, Strutinsky [97] first noticed that the difference in the number of stationary collective states in the ground state and at the saddle point was erroneously ignored by Bohr and Wheeler. Hence, he suggested that the fission-decay width derived from the Bohr-Wheeler transition-state theory should be multiplied by

$$
S=\frac{\hbar \omega_{\mathrm{gs}}}{T_{\mathrm{gs}}}
$$

where $\hbar \omega_{\mathrm{gs}}$ denotes the potential curvature in the ground state and is taken to be $1.0 \mathrm{MeV}$. $T_{\mathrm{gs}}$ stands for the nuclear temperature within the Fermi-gas model. Finally, the new combined fission-decay width is given by

$$
\Gamma_{f}=K \cdot S \cdot \Gamma_{f}^{\mathrm{BW}} .
$$

\subsection{Fission barrier}

The fission-barrier height entering Eq. (66) can be approximated as

$$
B_{f}=B_{\mathrm{LDM}}-\Delta E_{\mathrm{sh}},
$$

where $B_{\mathrm{LDM}}$ is the liquid-drop fission barrier and $\Delta E_{\mathrm{sh}}$ the ground-state shell-correction energy. It is assumed here that $\Delta E_{\text {sh }}$ practically vanishes at the saddle point as a consequence of the so-called topographical theorem [98].

\subsubsection{Shell-correction energy}

By default, the shell-correction energy is taken from the Möller-Nix table [15]. In addition, a correction factor $f$ is attached to it, namely

$$
\Delta E_{\mathrm{sh}}=f \cdot \Delta E_{\mathrm{MN}}
$$

The introduction of this factor was motivated by the fact that large uncertainties still remain in the shell-correction energy, which is crucially important for describing the synthesis of super-heavy nuclei. Since the liquid-drop barrier gradually vanishes with atomic number, the shell-correction energy should be responsible for the extra stability of super-heavy nuclei. Hence, a small variation in this correction factor (or in the fission barrier) could lead to a significant change in the final results (cf. Section 7). Note that the default value of $f$ was kept fixed at 1.0 .

In the literature, there also exist other theoretical models based on either the microscopic or macroscopic approaches [99-103]. To examine different models, the code can directly import a user-defined data file with the help of the option provided in the input file. Special care should be taken to guarantee the consistency between the liquid-drop fissionbarrier parameterization and the corresponding shell-correction energy.

\subsubsection{Thomas-Fermi model}

Two parameterizations of $B_{\mathrm{LDM}}$ based upon the Möller-Nix table have been incorporated into the KEWPIE2 code. The first one, which was developed within the framework of the Thomas-Fermi (TF) model [104], is simply written as

$$
B_{\mathrm{TF}}=P \cdot F(X),
$$

where the factor $P=A_{C}^{2 / 3}\left(1-k_{s} I_{C}^{2}\right)$ is related to the nuclear surface energy, and the coefficient of isospin dependence $k_{s}$ is defined as $1.9+\left(Z_{C}-80\right) / 75$. The fission parameter $X$ is given by

$$
X=\frac{Z_{C}^{2}}{A_{C}\left(1-k_{s} I_{C}^{2}\right)} \text {. }
$$

As regards the function $F(X)$, the explicit expression was obtained by fitting the experimental liquid-drop barriers for about 120 nuclei covering the pre-actinide and actinide regions,

$$
F(X)=\left\{\begin{array}{cc}
0.595553-0.124136\left(X-X_{1}\right), & \text { for } 30 \leq X \leq X_{1}, \\
1.99749 \cdot 10^{-4}\left(X_{0}-X\right)^{3}, & \text { for } X_{1} \leq X \leq X_{0},
\end{array}\right.
$$


where $X_{0}$ and $X_{1}$ are taken to be 48.5428 and 34.15 , respectively.

As mentioned in Ref. [89], Eq. (73) would provide a fairly good description of the experimental fission barriers for preactinides. In the case of heavier nuclei, it could lead to an underestimation of measured values. In the KEWPIE2 code, the Thomas-Fermi model has been chosen to be the default option for estimating liquid-drop fission barriers.

\subsubsection{Empirical fission-barrier heights}

The second formula was recently obtained using the LublinStrasbourg Drop (LSD) model [105]. Within this framework, the liquid-drop fission barrier can be well approximated by a Gaussian function, namely

$$
B_{\mathrm{LSD}}=B_{\max } \exp \left[-\left(\frac{I_{C}-I_{0}}{\Delta I}\right)^{2}\right]
$$

with

$$
\begin{aligned}
B_{\max } & =a_{0}+a_{1} Z_{C}+a_{2} Z_{C}^{2} 10^{-2}+a_{3} Z_{C}^{3} 10^{-4}, \\
I_{C} & =\left(A_{C}-2 Z_{C}\right) / A_{C} \\
I_{0} & =a_{4}+a_{5} Z_{C} 10^{-4} \\
\Delta I & =a_{6}+a_{7} Z_{C} 10^{-2}+a_{8} Z_{C}^{2} 10^{-4} .
\end{aligned}
$$

As for the parameters involved in this parameterization, their best-fit values can be found in Ref. [105].

It should be mentioned that the above two parameterizations for the liquid-drop fission barrier are both based on the MöllerNix table [15].

\section{Nuclear level density}

\subsection{Intrinsic state-density formula}

From the previous sections, it can be seen that the state density has a crucial role to play in describing the de-excitation of excited nuclei. In the code, an improved state-density formula, which was first proposed in Ref. [106], has been employed to estimate various decay widths. Compared to the simple FermiGas model, the advantage of introducing such a closed-form formula is that the divergence issue in vicinity of the origin can be nicely solved (cf. Fig. 3). In this subsection, for the sake of completeness, the state-density formula and some relevant physical quantities are briefly summarized.

The intrinsic state-density formula for a nucleus composed of two kinds of particles, namely protons and neutrons, is explicitly given by

$$
\begin{aligned}
\rho_{\text {int }}\left(E^{*}\right) & =\frac{\sqrt{\pi}}{12} \frac{\exp \left(\beta_{0} E^{*}+a / \beta_{0}\right)}{\sqrt{\beta_{0} E^{* 3}}}\left(\frac{g_{0}^{2}}{4 g_{n} g_{p}}\right)^{1 / 2} \\
& \times \frac{1-\exp \left(-a / \beta_{0}\right)}{\left[1-\frac{1}{2} E^{*} \beta_{0} \exp \left(-a / \beta_{0}\right)\right]^{1 / 2}}
\end{aligned}
$$

where $g_{n}$ and $g_{p}$ are respectively the neutron and proton singleparticle state densities at the Fermi energy. Here, $g_{0}=g_{n}+$

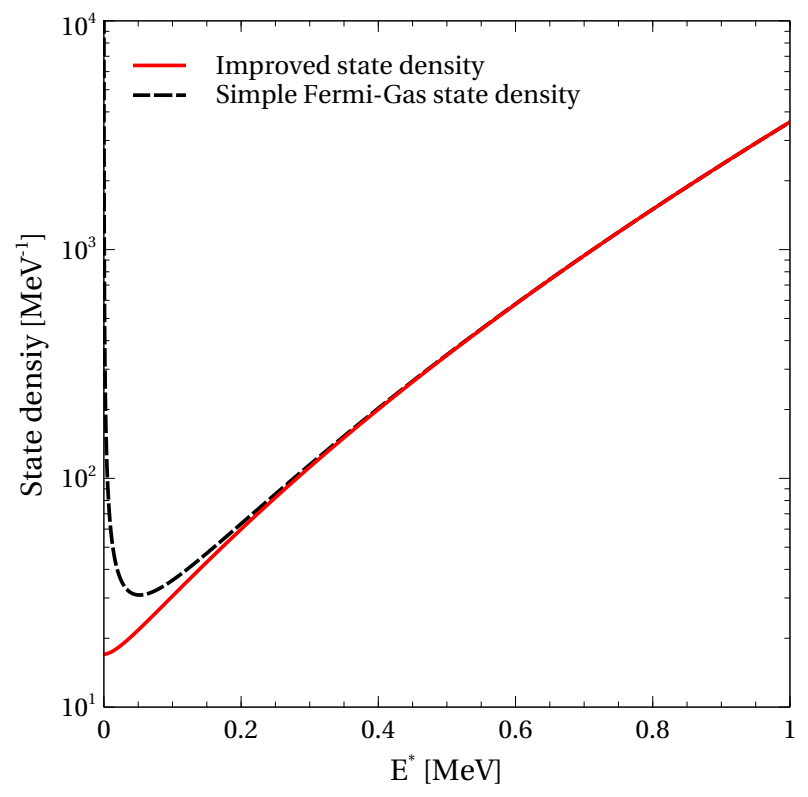

Fig. 3. Comparison of Eq. (78) with the simple Fermi-Gas state-density formula (cf. Eq. (81)). The divergence at energies close to the origin has been well eliminated.

$g_{p}$ and typically, one has the following approximation: $g_{n} \simeq$ $g_{p}$. In Eq. (78), $a$ refers to the nuclear level-density parameter that will be discussed later. It should be noted that the implicit relationship between $\beta_{0}$ and $E^{*}$ is expressed as

$$
\left(\frac{a}{\beta_{0}}\right)^{2}=\left(a E^{*}\right)\left[1-\exp \left(-\frac{a}{\beta_{0}}\right)\right] \text {. }
$$

When the excitation energy tends to zero $\left(a E^{*} \ll 1\right.$ or $a / \beta_{0} \ll$ 1 ), one simply has the limit $\beta_{0} \rightarrow 1 / E^{*}$, which leads to

$$
\rho_{\text {int }} \rightarrow \frac{1}{12} \sqrt{2 \pi} e a \exp \left(a E^{*}\right)
$$

where $e$ is the base of the natural logarithm. At higher excitation energies $\left(a E^{*} \gg 1\right.$ or $\left.a / \beta_{0} \gg 1\right)$, one obtains the well-known textbook formula,

$$
\rho_{\text {int }} \rightarrow \frac{\sqrt{\pi}}{12} \frac{\exp \left(2 \sqrt{a E^{*}}\right)}{a^{1 / 4} E^{* 5 / 4}},
$$

when $\beta_{0} \rightarrow \sqrt{a / E^{*}}=1 / T$ with $T$ being the nuclear temperature.

Eq. (78) is used within the framework of the WeisskopfEwing evaporation model. Regarding the Hauser-Feshbach formalism, the angular-momentum dependence of Eq. (78) should be considered. In this case, the state density with a fixed angular momentum is given by [107]

$$
\rho_{\text {int }}\left(E^{*}, J\right)=\frac{2 J+1}{\sqrt{2 \pi} \sigma_{\perp}^{3}} \exp \left[-\frac{(J+1 / 2)^{2}}{2 \sigma_{\perp}^{2}}\right] \rho_{\text {int }}\left(E^{*}\right) .
$$

where $\sigma_{\perp}^{2}$ is the spin cut-off parameter for axially deformed nuclei. It can be expressed as a function of the temperature $T$, namely

$$
\sigma_{\perp}^{2}=\frac{\mathfrak{J}_{\perp} T}{\hbar^{2}},
$$


where $\mathfrak{J}_{\perp}$ denotes the rigid-body moment of inertia perpendicular to the symmetry axis (cf. Subsection 6.3). It should be noted that Eq. (82) would be considered to result from the following approximation: $\rho_{\text {int }}\left(E^{*}, J\right) \propto \rho_{\text {int }}\left(E^{*}-E_{\text {rot }}\right) \sim$ $\rho_{\text {int }}\left(E^{*}\right) \exp \left(-E_{\text {rot }} / T\right)$, which is based on that fact that, for any nucleus with total excitation energy $E^{*}$ and angular momentum $J$, only $E^{*}-E_{\text {rot }}$ would be responsible for internal excitation. We would like to recall in this regard that the rotational energy $E_{\text {rot }}$ is generally defined as $J(J+1) /\left(2 \mathfrak{J}_{\perp}\right) \simeq(J+1 / 2)^{2} /\left(2 \mathfrak{J}_{\perp}\right)$. Formal derivation can be made following the standard statistical approach [107].

\subsection{Level-density parameter}

\subsubsection{Diffuse Thomas-Fermi model}

The default parameterization of the level-density parameter has been taken from Ref. [108]. Within the extended ThomasFermi model, the level-density parameter for a given nucleus is written as

$$
a=\frac{A}{14.61}\left(1+3.114 \frac{\mathfrak{B}_{s}}{A^{1 / 3}}+5.626 \frac{\mathfrak{B}_{k}}{A^{2 / 3}}\right)\left(1-\frac{I^{2}}{9}\right),
$$

where $I$ is the relative neutron excess and the surface term $\mathfrak{B}_{s}$ and the curvature term $\mathfrak{B}_{k}$ are respectively given as follows [109]

$$
\begin{aligned}
& \mathfrak{B}_{s}=1+\frac{2}{5} \alpha_{2}^{2}-\frac{4}{105} \alpha_{2}^{3}-\frac{66}{175} \alpha_{2}^{4}, \\
& \mathfrak{B}_{k}=1+\frac{2}{5} \alpha_{2}^{2}+\frac{16}{105} \alpha_{2}^{3}-\frac{82}{175} \alpha_{2}^{4} .
\end{aligned}
$$

In the ground state, $\alpha_{2}=\sqrt{5 / 4 \pi} \beta_{2}$, where $\beta_{2}$ refers to the quadrupole deformation parameter for the ground state. At the saddle point, we adopt the following expression $[109,110]$ :

$$
\alpha_{2}=\frac{7}{3} y-\frac{938}{765} y^{2}+9.499768 y^{3}-8.050944 y^{4},
$$

where $y=1-x$. According to Ref. [111], the fissility parameter $x$ can be parameterized as

$$
x=\frac{Z^{2}}{49.22 A\left(1-0.3803 I^{2}-20.489 I^{4}\right)} .
$$

Eq. (86) would only be valid for $x$ close to unity. It should be mentioned that, when the deformation parameter for the saddle point becomes smaller than that for the ground state, we simply add them together to redefine the quadrupole deformation of the saddle point. This ansatz can guarantee that the fission barrier shall always lie beyond the ground-state configuration.

\subsubsection{Empirical model}

A second option for the level-density parameter parameterization included in the code is due to Reisdorf [43]. Using the leptodermous approximation within the Thomas-Fermi model, one obtains the following closed-form formula:

$$
a=A\left(0.04543 r_{0}^{3}+0.1355 r_{0}^{2} \frac{\mathfrak{B}_{s}}{A^{1 / 3}}+0.1426 r_{0} \frac{\mathfrak{B}_{k}}{A^{2 / 3}}\right),
$$

where $\mathfrak{B}_{s}$ and $\mathfrak{B}_{k}$ are respectively the surface and curvature terms, as previously discussed. Here, $r_{0}$ has been determined to be $1.153 \mathrm{fm}$ by fitting to the measured values [43].

\subsubsection{Microscopic model}

The last one was recently proposed by Nerlo-Pomorska et al. [112]. Finite-temperature macroscopic-microscopic calculations were performed with the Yukawa folded mean field for 134 spherical even-even nuclei and 6 deformed ones at temperatures $0 \leq T \leq 5 \mathrm{MeV}$. The estimates for the level-density parameter obtained for different deformations are fitted by a liquid-drop type formula, which is expressed in the following form:

$$
a=0.092 A+0.036 A^{2 / 3} \mathfrak{B}_{s}+0.275 A^{1 / 3} \mathfrak{B}_{k}-0.00146 \frac{Z^{2}}{A^{1 / 3}} \mathfrak{B}_{c},
$$

where the new term $\mathfrak{B}_{c}$ is the Coulomb term for a deformed nucleus, which is given as follows [109]:

$$
\mathfrak{B}_{c}=1-\frac{1}{5} \alpha_{2}^{2}-\frac{4}{105} \alpha_{2}^{3}+\frac{51}{245} \alpha_{2}^{4} .
$$

It would be worth mentioning that, based upon the microscopic model, Eq. (89) predicts a bit smaller values compared to those coming form the diffuse Thomas-Fermi model.

\subsection{Moment of inertia}

Let us now consider a axially-symmetrical deformed nucleus, whose moment of inertia perpendicular to the symmetry axis is given as follows [113]:

$$
\mathfrak{J}_{\perp}^{\mathrm{gs}}=\frac{2}{5} M_{A} R_{A}^{2}\left(1+\sqrt{\frac{5}{16 \pi}} \beta_{2}+\frac{45}{28 \pi} \beta_{2}^{2}\right),
$$

where $M_{A}$ and $R_{A}$ are the nuclear mass and the radius of the spherical shape, respectively.

At the saddle point, one only needs to replace the groundstate deformation parameter appearing in the above expression by the saddle-point one (cf. Eq. (86)) to obtain $\mathfrak{J}_{\perp}^{\text {sd }}$.

It should be noted that, as shown in many experimental studies [114], the measured values of the inertia moment are usually lower than those estimated within the rigid-body model. This discrepancy has been corrected by multiplying $\mathfrak{J}_{\perp}^{\mathrm{gs}}$ and $\mathfrak{J}_{\perp}^{\text {sd }}$ by a constant factor having a default value of 0.4 . It can be adjusted by the user.

\subsection{Ignatyuk's prescription}

It has been realized for many years that the shell-correction effects on the level density parameter gradually drops with increasing excitation energy. To account for this damping phenomena, we adopt the so-called Ignatyuk's prescription [115], which assumes that the level-density parameter would be regarded as a function of the excitation energy. In the ground state, one has the following explicit expression:

$$
a_{\mathrm{gs}}\left(E^{*}\right)=a\left[1+\left(1-e^{-E^{*} / E_{d}}\right) \frac{\Delta E_{\mathrm{sh}}}{E^{*}}\right],
$$

where the default value of the shell-damping energy $E_{d}$ has been arbitrarily fixed at $19.0 \mathrm{MeV}$, which can be adjusted by the user. 


\subsection{Collective enhancement factor}

Based upon the adiabatic formalism, where the internal and collective degrees of freedom are completely decoupled from each other, the intrinsic state density should be enhanced by a certain factor as a function of excitation energy $E^{*}$, namely

$$
\rho\left(E^{*}, J\right)=\rho_{\text {int }}\left(E^{*}, J\right) \kappa_{\text {coll }}\left(E^{*}\right) .
$$

The collective enhancement effect would quickly falls with increasing excitation energy. This can be interpreted in a way that, at higher energies, the fluctuations in the nuclear deformation could become larger due to single-particle motions. As a consequence, the collective and internal degrees of freedom begin to interfere with each other. This would lead to an absorption of the collective states into the intrinsic ones. More concretely, one should have $\kappa_{\text {coll }}\left(E^{*}\right) \rightarrow 1$ when $E^{*}$ goes beyond a certain critical energy $E_{\mathrm{cr}}$.

In Ref. [39], Junghans et al. investigated the following formulation for the collective enhancement factor:

$$
\kappa_{\text {coll }}\left(E^{*}\right)=\left\{\begin{array}{cl}
\left(\sigma_{\perp}^{2}-1\right) f\left(E^{*}\right)+1, & \text { for } \sigma_{\perp}^{2}>1, \\
1, & \text { for } \sigma_{\perp}^{2} \leq 1,
\end{array}\right.
$$

in which the spin cut-off parameter $\sigma_{\perp}^{2}$ is given by

$$
\sigma_{\perp}^{2}=\left\{\begin{array}{cc}
\frac{\mathfrak{J}_{\perp} T}{\hbar_{\mathfrak{J}}^{2}}, & \text { when }\left|\beta_{2}\right|>0.15 \text { for rotations, } \\
S \Theta^{2} \frac{\mathfrak{J}_{\perp} T}{\hbar^{2}}, & \text { when }\left|\beta_{2}\right| \leq 0.15 \text { for vibrations, }
\end{array}\right.
$$

where $\beta_{2}$ is the quadrupole-deformation parameter and $\Theta=$ $0.022+0.003 \Delta N+0.005 \Delta Z$ a dynamical deformation parameter that somehow accounts for the variation of vibrational energy levels as a function of the distance from the closed shell. The cut-off factor $f\left(E^{*}\right)$ is taken to be $1 /\left\{1+\exp \left[\left(E^{*}-E_{\mathrm{cr}}\right) / d_{\mathrm{cr}}\right]\right\}$ with $E_{\mathrm{cr}}=40 \mathrm{MeV}$ and $d_{\mathrm{cr}}=10 \mathrm{MeV} . S$ stands for a free parameter, whose optimum value was determined to be 25 [39]. Eq. (95) shows that the collective character of the nuclear motion can be described as a function of the quadrupole deformation parameter $\beta_{2}$ of the nuclear shape: the rotation dominates the collective motion of nuclei when $\left|\beta_{2}\right|>0.15$ (deformed nuclei), whereas the vibration intervenes for $\left|\beta_{2}\right| \leq 0.15$. To some extent, it seems as if the rigid-body moment of inertia $\mathfrak{J}_{\perp}$ describing the rotational motion of deformed nuclei is gradually merged into the irrational-flow moment of inertia, namely $\mathfrak{J}_{\perp}^{\text {irr }}=\Theta^{2} \mathfrak{J}_{\perp}$, which would be more appropriate for describing nuclear vibrations.

In Ref. [116], on the basis of Junghans et al.'s results, Zagrebaev et al. proposed an unified expression for the collective enhancement factor, which gives a smooth transition between $\mathfrak{J}_{\perp}^{\text {irr }}$ and $\mathfrak{J}_{\perp}^{\text {rig }}$. It is simply given by

$$
\kappa_{\mathrm{coll}}\left(E^{*}\right)=\kappa_{\mathrm{rot}}\left(E^{*}\right) \varphi\left(\beta_{2}\right)+\kappa_{\mathrm{vib}}\left(E^{*}\right)\left[1-\varphi\left(\beta_{2}\right)\right],
$$

with

$$
\varphi\left(\beta_{2}\right)=\left[1+\exp \left(\frac{\beta_{2}^{0}-\left|\beta_{2}\right|}{\Delta \beta_{2}}\right)\right]^{-1},
$$

where $\beta_{2}^{0}=0.15$ and $\Delta \beta_{2}=0.04$. This formula has been implemented in the current version of the code.

\subsection{Pairing effect}

The pairing effect can approximately be taken into account by replacing $E^{*}$ by $E^{*}-\Delta_{\text {pair }}$ in the above expressions. Following [117], it is simply given by

$$
\Delta_{\text {pair }}=\left\{\begin{array}{cc}
0, & \text { if } Z \text { and } N \text { are both odd, } \\
12 / \sqrt{A}, & \text { if } A \text { is odd, } \\
24 / \sqrt{A}, & \text { if } Z \text { and } N \text { are both even. }
\end{array}\right.
$$

As will be seen in the next section, this correction to the excitation energy would be necessary for the reproduction of experimental results.

\section{Computational examples and sensibility analysis}

In this section, some typical applications of the KEWPIE2 code are demonstrated. It is commonly known that the usefulness of any theoretical model essentially depends upon the accuracy and reliability of its final outcomes. However, since all models are not perfect, the exact input data are rarely, if ever available, which means that their theoretical outcomes would always be uncertain to some extent. This is the main reason why a sensibility analysis would be needed in order to understand the impact of both model and parameter uncertainties on the final results.

Among all the input data comprised in the code, we are mainly focusing on three quantities, that is, the reduced friction coefficient $\beta$, the shell-damping energy $E_{d}$ and the fission barrier $B_{f}$, which play a major role in theoretical calculations [6]. The first two are free parameters, whereas the third one is thought to be model-dependent. For the sake of clarity, their typical ranges are summarized as follows:

- The reduced friction parameter $\beta \sim 1.0-9.0 \mathrm{zs}^{-1}[38,118]$.

- The shell-damping energy $E_{d} \sim 13.0-25.0 \mathrm{MeV}[115$, 119].

- The fission-barrier heights could differ by 1 or $2 \mathrm{MeV}$ from each other $[104,105,120]$.

As a starting point, we adopt the following default options: $\beta=5.0 \mathrm{zs}^{-1}, E_{d}=19.0 \mathrm{MeV}$ and the fission barrier is estimated using the Thomas-Fermi model (cf. Eq. (73)). In the following study, we keep using these default options, except that the use of other values is stated. Moreover, different theoretical approaches, such as the Kramers-Strutinsky correction to the fission-decay width and collective enhancement factor, might also affect the simulation results.

For comparison with experimental data, we mainly concentrate on the reaction systems where the fusion hindrance might not be present in order to avoid some remaining theoretical ambiguities related to this phenomenon. In this sense, the fusion probability simply corresponds to the capture one. In addition, the Weisskopf-Ewing model is employed to estimate the particle-evaporation width. As has been carefully tested, this approach can be regarded as a good approximation of the Hauser-Feshbach formalism that is usually quite timeconsuming. It should be also mentioned that the reaction systems selected in this section (lying within a wide energy range) 


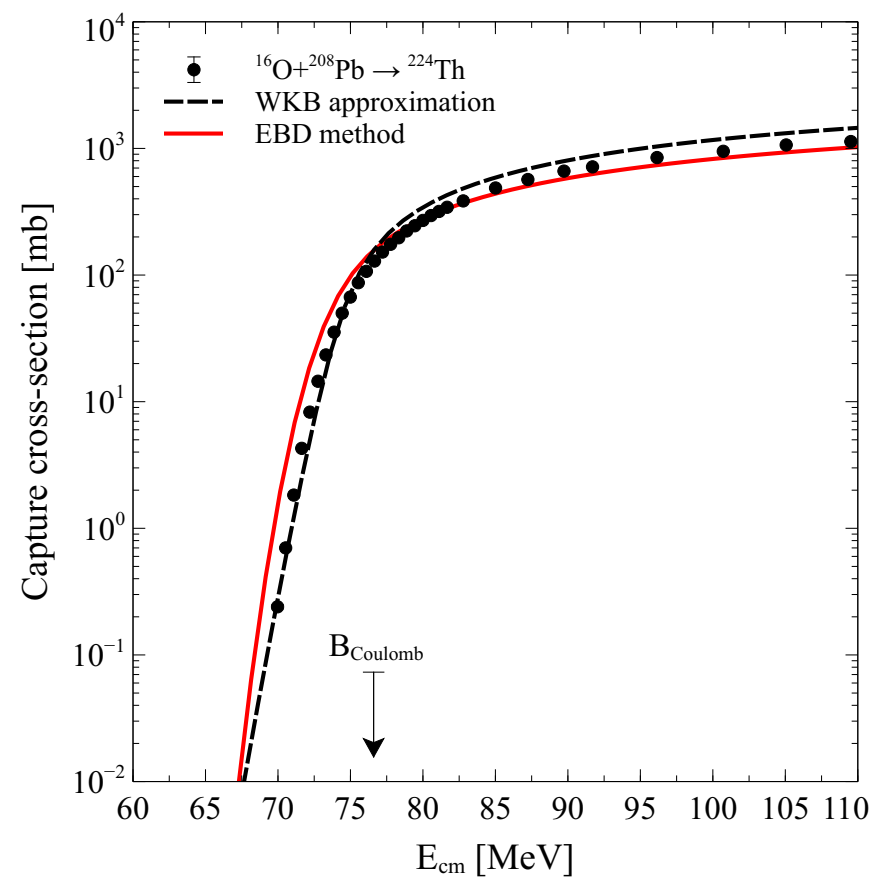

Fig. 4. Comparison of the estimated capture cross-sections with experimental measurements. The solid symbols refer to the experimental data taken from Ref. [63]. The WKB approximation (dashed black curve) and the EBD method (solid red curve), have been employed for computing fusion excitation functions. The Coulomb barrier is estimated to be about $76 \mathrm{MeV}$ (indicated by the black arrow).

would allow us to take a closer look at the effects of model uncertainties on the calculated results.

\subsection{Reaction ${ }^{208} \mathrm{~Pb}\left({ }^{16} \mathrm{O}, x \mathrm{n}\right)^{224-x} \mathrm{Th}$}

First of all, we would like to investigate this well-measured fusion-evaporation reaction, where $x$ equals 2,3 and 4 .

Fig. 4 displays the calculated capture cross-sections, which are systematically compared with the available experimental data. As already mentioned in Section 3, KEWPIE2 comprises two basic approaches, namely the WKB approximation and empirical barrier-distribution method (EBD), to estimate the capture cross-section. The calculated results appear quite close to each other at both low and high incident energies. It is readily seen that the EBD method seems able to reproduce the general trend of the experimental data at higher energies, whereas an evident overestimation of the capture cross-section has been observed below the Coulomb barrier. To understand this issue, it would be helpful to recall that the EBD approach is mainly based upon the assumption that fusion barrier is normally distributed. (The idea is somewhat similar to the coupled-channels approach where each coupling term results in an effective fusion barrier.) Theoretical calculations at sub-barrier energies are usually quite sensitive to the barrier parameter $w$, whose parameterization (cf. Eq. (29)) still remains rather crude. (The uncertainty associated with the width parameter can reach up to about 1.0 MeV [46].) This might be the reason why the EBD method tends to overestimate the measured values at energies below the Coulomb barrier. Note that the logarithmic scale has

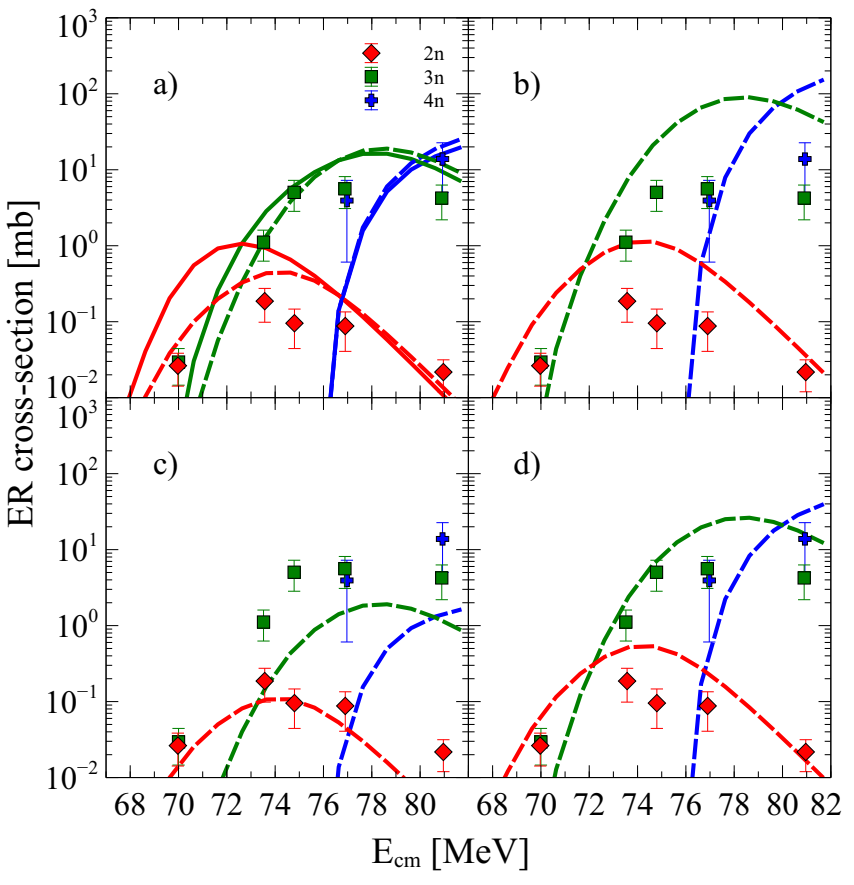

Fig. 5. Comparison of the estimated ER excitation functions for the reaction ${ }^{208} \mathrm{~Pb}\left({ }^{16} \mathrm{O}, x \mathrm{n}\right){ }^{224-x} \mathrm{Th}$. The solid symbols represent the experimental data taken from Ref. [121]. The calculated ER cross-sections of 2n-, 3n- and $4 n$-channels are respectively shown by the red, green and blue curves. The solid curves correspond to the calculations based upon the EBD method and dashed curves to those performed within the WKB approximation. Note that the WKB approximation has been chosen to calculate the capture cross-section. In Figs. 5(b), (c) and (d), we compare the computational results obtained without considering the collective-enhancement factor (b), without considering the Kramers-Strutinsky correction factor (c) and without considering both of them (d).

been employed here, the calculated capture cross-sections can even differ by less than one order of magnitude at sub-barrier energies. Accordingly, special care should always be given to the comparison between theoretical calculations and measurements.

Regarding the evaporation process, $2 n-, 3 n$ - and 4n-channels have been investigated within the Weisskopf-Ewing model. Fig. 5(a) compares the estimated ER excitation functions with the available experimental data. It is clearly observed that the excitation function based upon the EBD method is largely enhanced at low energies compared to the WKB approximation. This is essentially due to the overestimation of the capture cross-section at sub-barrier energies. As a whole, the experimental data can be reproduced rather well be means of the WKB approximation, especially for the $2 n$ - and $3 n$-channels, whose peak positions or optimum energies are found to be in nice agreement with the measurements. This is because the neutron-separation energies are estimated from the available experimental masses. Hence, we adopt the WKB approximation to estimate the ER excitation functions for the reaction ${ }^{208} \mathrm{~Pb}\left({ }^{16} \mathrm{O}, x \mathrm{n}\right){ }^{224-x} \mathrm{Th}$.

Figs. 5(b)-(d) illustrate the impact of modeling uncertainties on the ER excitation functions. Here, we mainly address two interesting factors, namely Kramers-Strutinsky correction factor 


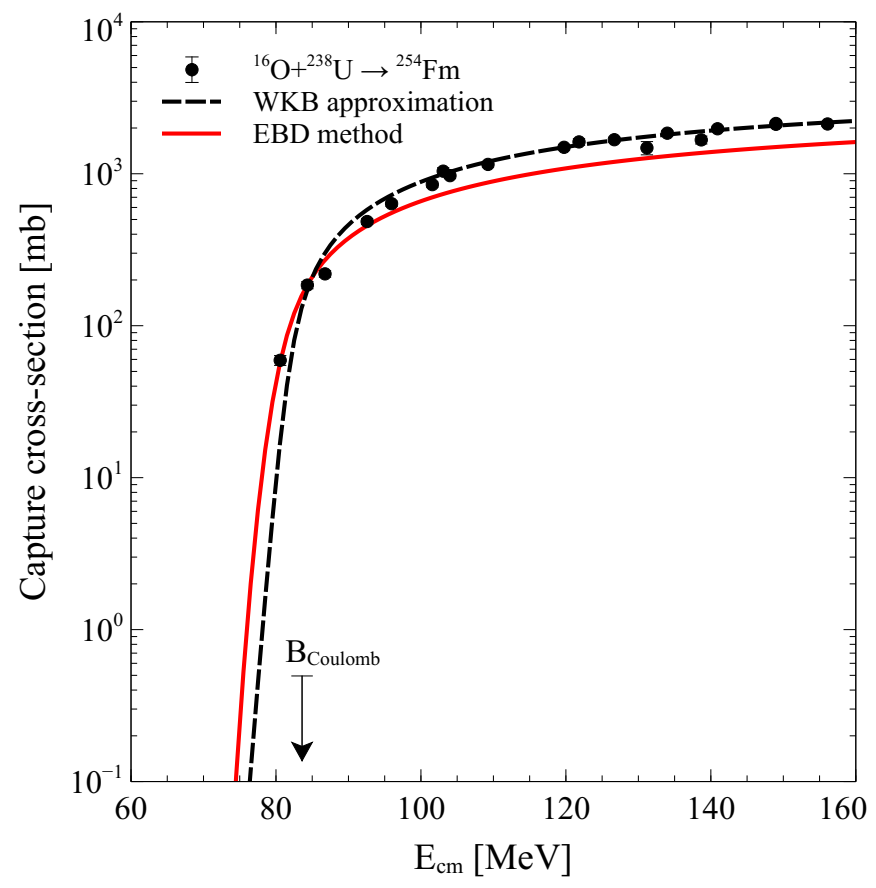

Fig. 6. Same as Fig. 4, but for the fusion-evaporation reaction ${ }^{238} \mathrm{U}\left({ }^{16} \mathrm{O}\right.$, $x \mathrm{n})^{254-x} \mathrm{Fm}$. The solid symbols represent the experimental data taken from Refs. [123-125]. The Coulomb barrier is estimated to be about $84 \mathrm{MeV}$ (indicated by the black arrow).

and collective-enhancement factor. As previously mentioned, the former takes into account the influence of nuclear viscosity on the fission process and the latter is responsible for correcting the state density due to low-lying collective states. In the literature, some authors employed both of them [20, 116, 122], while others none of them [19]. The computational results obtained without considering one or both of them have been clearly displayed in Figs. 5(b)-(d). The default parameter values are kept for all these calculations. It is clearly shown that, without considering the collective-enhancement factor, the maximum ER cross-sections seem to grow by a factor of about $3-6$, whereas they drop by a factor of about $4-15$ when taking out the Kramers-Strutinsky factor. Even more interesting is the fact that, when both of them are removed, again the computational results gradually approach the measured data. This might interpret why some simplified theoretical models without including both factors could also reproduce experimental data rather well.

\subsection{Reaction ${ }^{238} \mathrm{U}\left({ }^{16} \mathrm{O}, x \mathrm{n}\right){ }^{254-x} \mathrm{Fm}$}

The second example aims to study the following fusionevaporation reaction: ${ }^{238} \mathrm{U}\left({ }^{16} \mathrm{O}, x \mathrm{n}\right){ }^{254-x} \mathrm{Fm}$ with $x$ being 4,5 and 6 .

The capture cross-sections calculated using the EBD method and the WKB approximation are compared in Fig. 6. It seems that they are both able to reproduce the experimental data over the whole energy range. The EBD method slightly underestimates the capture excitation function at above-barrier energies, whereas it agrees rather well with the experimental data at subbarrier energies.

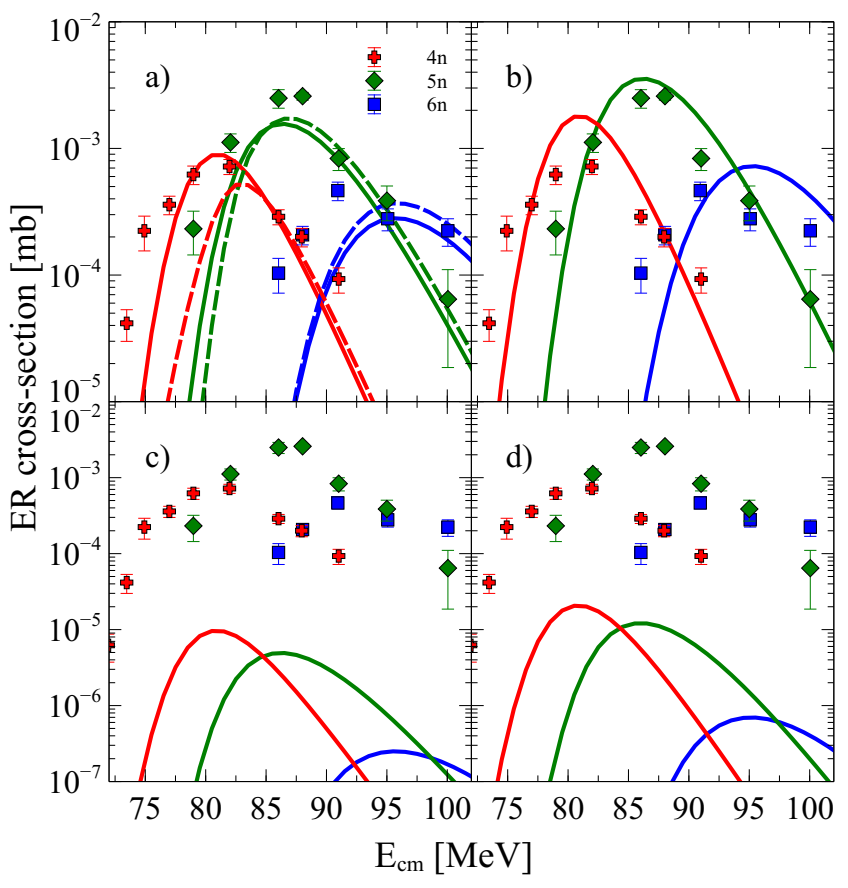

Fig. 7. Same as Fig 5, but for the reaction ${ }^{238} \mathrm{U}\left({ }^{16} \mathrm{O}, x \mathrm{n}\right){ }^{254-x} \mathrm{Fm}$. The solid symbols stand for the experimental data taken from Ref. [126]. The calculated ER cross-sections of 4n-, 5n- and 6n-channels are respectively shown by the red, green and blue curves. Note that the EBD method has been chosen to calculate the capture cross-section.

In Fig. 7(a), the ER cross-sections estimated with both capture models are compared with the experimental data for the 4n-, 5n- and 6n-channels. A dramatic underestimation at subbarrier energies has been confirmed in the case of the WKB approximation. However, using the EBD method, the calculated excitation functions are found to be in nice agreement with the measured data. Here, the default parameter values are kept for the present calculations.

Figs. 7(b)-(d) display the same model sensitivity analysis for the reaction ${ }^{238} \mathrm{U}\left({ }^{16} \mathrm{O}, x \mathrm{n}\right){ }^{254-x} \mathrm{Fm}$. Fig. 7(b) compares the theoretical calculations without accounting for the collective enhancement factor. The maximum values of the ER excitation functions appear to increase by a smaller factor compared to the previous reaction. This might be due to the fact that, as the excitation energy goes up, the collective effects would probably diminish in a gradual manner (cf. Section 6). Fig. 7(c) tells us that the estimated cross-sections are considerably reduced after taking out the Kramers-Strutinsky correction factor. The decrease in the maximum ER cross-section can even reach up to two or three orders of magnitude for 5- and 6n-channels. To interpret this large discrepancy, we should be aware that the probability of evaporating $s$ neutrons from a heavy nucleus would be roughly proportional to the product $\prod_{i=0}^{s-1}\left(\Gamma_{n} / \Gamma_{f}\right)_{i}$, or related to the $s$-th power of the Kramers-Strutinsky factor which only affects the calculation of $\Gamma_{f}$. It should be noted that the Kramers-Strutinsky correction factor is estimated to be of the order of 0.2 , so that the more neutrons evaporated, the more important its influence on the ratio of decay widths and thus on the neutron-evaporation probability. Moreover, the magnitude 


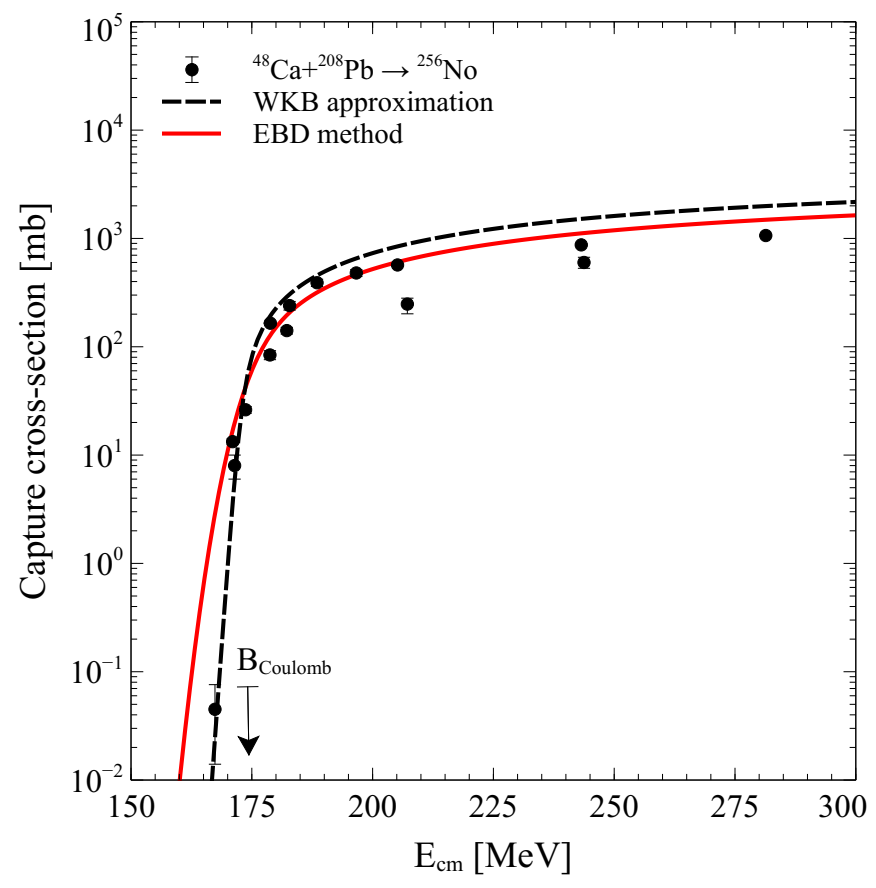

Fig. 8. Same as Fig. 4, but for the fusion-evaporation reaction ${ }^{208} \mathrm{~Pb}\left({ }^{48} \mathrm{Ca}\right.$, $x \mathrm{n})^{256-x}$ No. The solid symbols represent the experimental data taken from Refs. [128-130]. The Coulomb barrier is estimated to be about $175 \mathrm{MeV}$ (indicated by the black arrow).

of this correction factor also becomes lower with increasing excitation energy. Finally, Fig. 7(d) shows the result obtained without considering simultaneously these two factors. Hence, it would be crucially important for correctly modeling the competition between neutron emission and fission at high excitation energies. To this extent, the model uncertainty needs to be well considered when performing theoretical calculations [127].

\subsection{Reaction ${ }^{208} \mathrm{~Pb}\left({ }^{48} \mathrm{Ca}, x \mathrm{n}\right)^{256-x} \mathrm{No}$}

As a third example, we would like to investigate the reaction ${ }^{208} \mathrm{~Pb}\left({ }^{48} \mathrm{Ca}, x \mathrm{n}\right){ }^{256-x}$ No with $x$ being $1-3$, which is of special interest for experimentalists. For instance, a recent study concerning the measurement of the fission barrier in ${ }^{254} \mathrm{No}$ [133] has been performed by making use of this fusion-evaporation reaction. Furthermore, within the promising project SPIRAL2 at GANIL, the nuclear structure of ${ }^{254}$ No will be investigated by means of the same reaction [9].

The capture and evaporation processes have been separately examined. Fig. 8 shows the estimated fusion excitation functions based upon the two methods. Again, using the EBD method, a significant enhancement of the capture cross-section at sub-barrier energies has been observed in comparison with those obtained from the WKB approximation. It should be mentioned that the reaction ${ }^{208} \mathrm{~Pb}\left({ }^{48} \mathrm{Ca}, x \mathrm{n}\right){ }^{256-x}$ No might lie on the border between the regions with and without the fusion hindrance, or in other words, the formation probability $P_{\text {form }}$ could be slightly less than unity.

The estimated ER cross-sections for the 1n-, 2n- and 3nchannels are shown in Fig. 9(a) and a comparison with the available experimental measurements has also been performed. In

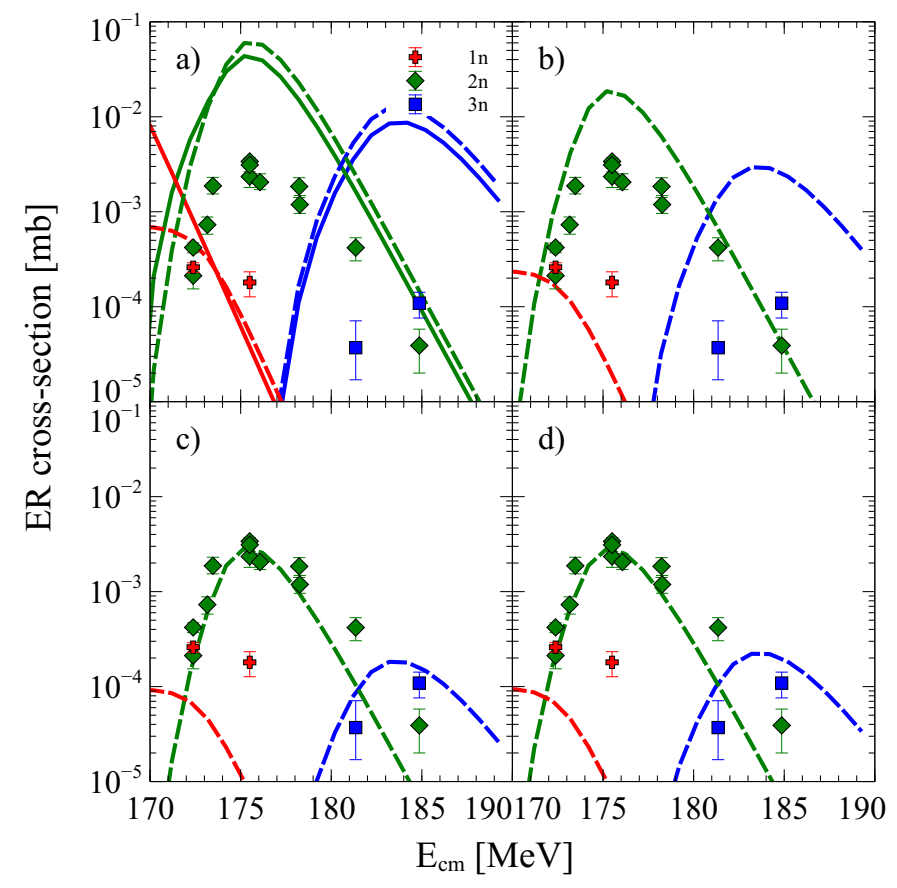

Fig. 9. Comparison of the estimated ER excitation functions for the reaction ${ }^{208} \mathrm{~Pb}\left({ }^{48} \mathrm{Ca}, x \mathrm{n}\right){ }^{256-x}$ No. The solid symbols stand for the experimental data taken from Refs. [131, 132]. The estimated ER cross-sections of 1n-, 2n- and $3 n$-channels are respectively shown by the red, green and blue curves. The solid curves correspond to the calculations based upon the EBD method and the dashed curves to those performed within the WKB approximation. Note that the WKB approximation has been chosen to estimate the capture cross-section in Figs. 9(b), (c) and (d), where the estimated ER cross-sections are based upon the LSD fission-barrier model with the default parameter values (b), decreasing the value of $\beta$ by about $80 \%$ (c), decreasing the value of $E_{d}$ by about $30 \%$ (d).

both cases, it is clearly shown that the calculated ER excitation functions are systematically higher compare to the measured data. To give a reasonable fit, we first adopt the WKB approximation for estimating the capture cross-section and employ the other fission-barrier model incorporated into the KEWPIE2 code, which was recently developed by means of the LSD model [105]. In Fig. 9(b), it is readily seen that, after changing the fission-barrier parameterization, the estimated excitation functions are getting closer to the data points. For the sake of clarity, the fission-barrier heights of the decaying isotopes are listed in Table 1. Overall, the LSD fission-barrier heights

Table 1

The liquid-drop fission-barrier heights of the nobelium isotopes estimated within the TF and LSD models. Here the shell-correction energies are taken from Ref. [15].

\begin{tabular}{ccccc}
\hline$Z$ & $A$ & $B_{\mathrm{TF}}[\mathrm{MeV}]$ & $B_{\mathrm{LSD}}[\mathrm{MeV}]$ & $\Delta_{\text {sh }}[\mathrm{MeV}]$ \\
\hline 102 & 253 & 0.38 & 0.10 & -4.49 \\
102 & 254 & 0.40 & 0.10 & -4.65 \\
102 & 255 & 0.41 & 0.11 & -4.44 \\
102 & 256 & 0.42 & 0.11 & -4.19 \\
\hline
\end{tabular}

appear a bit smaller than those based upon the TF model. Nevertheless, a considerable discrepancy still persists. Based upon the LSD fission-barrier model, we separately tune the reduced 


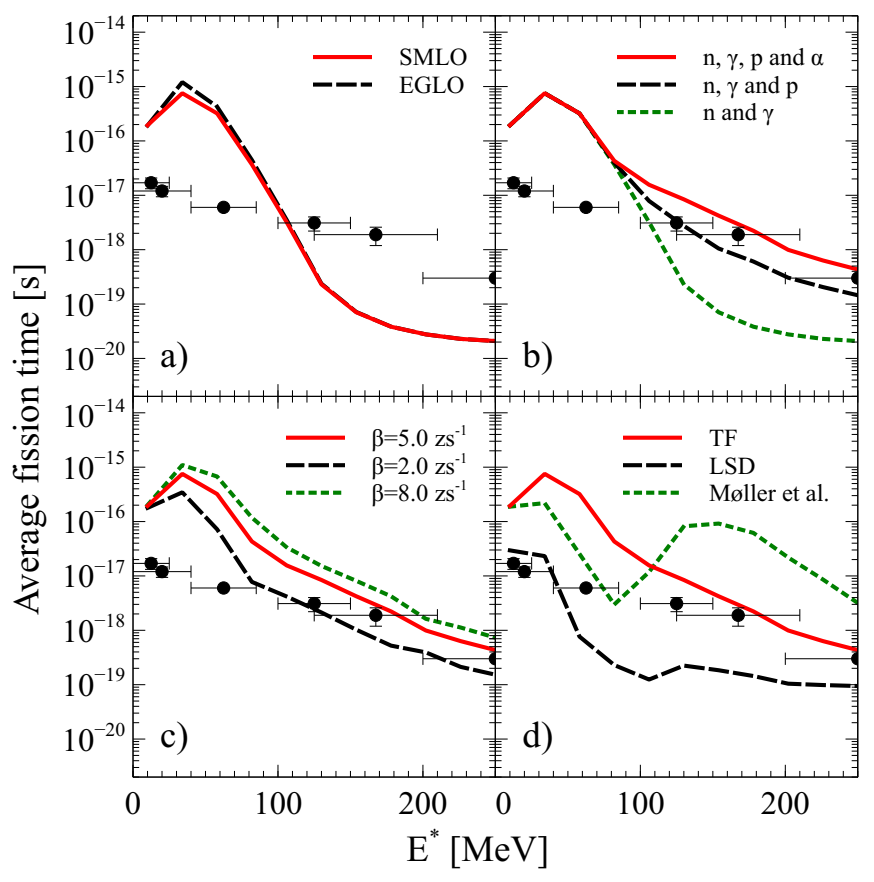

Fig. 10. Comparison of the calculated average fission time with the experimental measurements. The solid symbols stand for the experimental data taken from Refs. $[1,2]$. The calculated average fission times are shown by curves in Figs. 9(a), (b), (c) and (d). The solid red curves correspond to the calculations with default parameter values and the SMLO model for $\gamma$-ray emission. Other curves stand for the estimated results based upon the EGLO model (a), including the emission of charged particles (b), taking into account the different values of the reduced friction coefficient (charged particles included) (c), and using the different fission-barrier models (charged particles included) (d).

friction coefficient and the damping energy. The calculated results have been illustrated in Figs. 9(c) and 9(d). More concretely, with respect to their default values, we modify only one parameter at each time. To reproduce the measured data, the reduced friction parameter $\beta$ should be decreased by about $80 \%$ (down to $1.0 \mathrm{zs}^{-1}$ ) and $E_{d}$ decreased by about $30 \%$ (down to $13 \mathrm{MeV}$ ). As can be seen from this example, we can tune parameter values within a reasonable range and choose pertinent models to reproduce the data, even though the measured values appear to be far lower with respect to computational results based upon the default options. Furthermore, it should be also mentioned that the fusion hindrance might occur in this reaction system, that is, the capture cross-section could be reduced by introducing the formation probability $P_{\text {form }}$, which is, however, not considered in the present calculation because of some remaining ambiguities.

\subsection{Average fission time of uranium-238}

Up to now, the calculation of ER cross-sections has been well performed using the statistical part of the KEWPIE2 code, which is actually related to the long time limit of the population of residual nuclei. In this subsection, the dynamical part of the code, which is based upon the time-dependent Bateman equations (cf. Section 2), is employed to calculate the average fission time of ${ }^{238} \mathrm{U}$. The experiment was carried out many

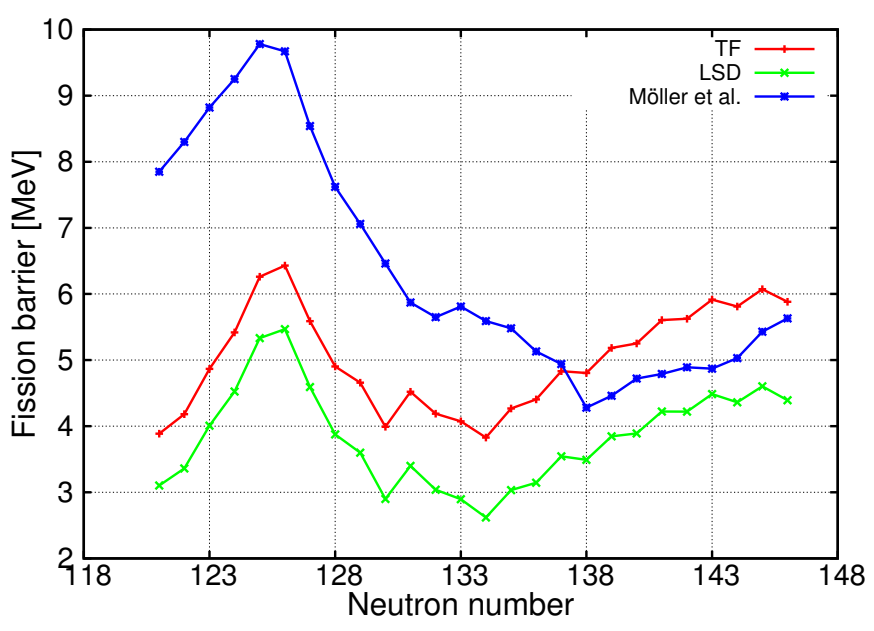

Fig. 11. Comparison of the total fission barriers of the uranium isotopes based upon different fission-barrier models (see text).

years ago at GANIL by making use of the crystal-blocking technique [1]. The measured data are plotted in Fig. 10.

First, let us only consider the competition between neutron evaporation, fission and $\gamma$-ray emission. The calculation was achieved with the default parameter values. Both the EGLO and SLMO models have been employed to estimate the radiation strength function. Fig. 10(a) shows that the calculated results overestimate the measured values at low excitation energies, whereas an underestimation has been recorded when the excitation energy goes beyond about $100 \mathrm{MeV}$. To resolve this discrepancy, it would be necessary to consider the emission of charged particles, which cannot be neglected at high excitation energies. As can be seen in Fig. 10(b), the emission of charged particles (protons and $\alpha$ particles) appears to have a great impact on the final results, but only affects the high-energy part of the spectrum.

The calculated average fission time would strongly depend on the fission-barrier height and the magnitude of the nuclear viscosity. Figs. 10(c) and 10(d) demonstrate how the reduced friction coefficient and the fission-barrier height affect the calculated average fission time (charged particles included). Regarding the dissipation effect, Fig. 10(c) tells us that the average fission time systematically grows with reduced friction coefficient due to the fact that fission is increasingly damped. As regards the fission-barrier model, Fig. 10(d) clearly shows that, with lower fission-barrier heights based upon the LSD model (cf. Fig. 11), the low-energy part of the spectrum dramatically falls and seems closer to the experimental data, whereas an underestimation of the high-energy part has been observed. The general trend of the curve corresponding to the Möller et al.'s model [120] seems quite strange. It gradually drops at low excitation energies but then starts to increase by orders of magnitude. This could be explained by the fact that, as shown in Fig. 11, the theoretical fission-barrier heights of the residual nuclei generally appear much higher compared to the TF and LSD fission-barrier models, so that more nuclei are expected to survive against fission.

The abnormal behavior (peak) of the calculated values at en- 
ergies around $20 \sim 60 \mathrm{MeV}$ still remains an open question to us. As discussed earlier in Ref. [5], this significant discrepancy between the theoretical calculations and experimental measurements might be due to the fact that, at low excitation energies, it has been experimentally observed that the asymmetric fission appears more favorable and thus the traditional Bohr-Wheeler theory would no longer be valid. In other words, the fissioning system could follow an asymmetric path rather than a symmetric one and thus other degrees of freedom shall come into play during the fission process. Another possible reason for this significant discrepancy is that the fission-barrier height is not precisely evaluated. As can be seen in Fig. 11, the estimated values might even differ by $3-4 \mathrm{MeV}$ from each other and thus cause a dramatic change in the final result. Moreover, it should be noted that the experimental error bars associated with excitation energies are extremely large so that the measured values might become less reliable. Anyhow, further consideration will be needed to overcome this delicate issue.

\section{Conclusion and perspectives}

In summary, the KEWPIE2 code has been carefully tested and typical applications, such as the calculation of ER crosssections for heavy nuclei and the estimation of the average fission time for uranium-238, have been shown. Compared to its previous version, the values of some model parameters have been reasonably corrected and a better agreement with experimental data was verified. Furthermore, according to the sensibility analysis, it has been well established that uncertainties coming from both parameters and models are generally essential for theoretical predictions and therefore should be investigated in a more formal manner. For instance, the calculated ER cross-sections and average fission time seem to be crucially sensitive to the uncertainty associated with fission-barrier heights, which could give rise to a remarkable change (up to orders of magnitude) in the final outcomes. To provide a more accurate description of the fusion-evaporation reaction, it would be necessary to perform a complete uncertainty analysis, which permits us to look more closely at the impact of uncertainty propagation on numerical modeling and thus to constrain the values of input parameters. Some follow-up work is currently underway and will be published elsewhere.

\section{Appendix A. Gauss-Legendre quadrature}

The numerical integration is quite time-consuming especially in the fusion and cascade calculations. One should somehow avoid the traditional discretization methods, such as the rectangle and trapezoidal rules, since they would imply a relatively large number of iterations. The Gauss-Legendre quadrature rule has been employed to evaluate the integral involved in the WKB approach. Recalling that, to calculate $\int_{a}^{b} f(x) \mathrm{d} x$, one has the following quadrature formula:

$$
\int_{a}^{b} f(x) \mathrm{d} x \simeq \frac{b-a}{2} \sum_{i=1}^{n} w_{i} f\left(\frac{b-a}{2} x_{i}+\frac{b+a}{2}\right),
$$

where $x_{i}$ refers to the $i^{\text {th }}$ root of the Legendre polynomial $P_{n}(x)$ and the weight $w_{i}$ is given by $2 /\left\{\left(1-x_{i}^{2}\right)\left[P_{n}^{\prime}\left(x_{i}\right)\right]^{2}\right\}$. In the code, the default value of $n$ has been chosen to be 32, which can be modified according to the precision requirement. The exact values of the weights and roots can be found in Ref. [134].

\section{Appendix B. Incoming-Wave Boundary Condition}

Within the optical model, the Schrödinger equation (cf. Eq. (48)) is commonly solved by imposing the following boundary conditions:

$$
\begin{aligned}
u(r) & \sim r^{l+1}, \text { for } r \rightarrow 0, \\
& \sim \frac{i}{2}\left[H_{l}^{(-)}(k r)-S_{l} H_{l}^{(+)}(k r)\right], \text { for } r \rightarrow+\infty,
\end{aligned}
$$

where $S_{l}$ denotes the S-matrix. $H^{(\mp)}$ are respectively related to the regular and irregular Coulomb wave functions, namely $F_{l}(\eta, k r)$ and $G_{l}(\eta, k r)$, by

$$
\begin{aligned}
& H_{l}^{(-)}=G_{l}(\eta, k r)-F_{l}(\eta, k r), \\
& H_{l}^{(+)}=G_{l}(\eta, k r)+F_{l}(\eta, k r),
\end{aligned}
$$

where $\eta$ is the so-called Sommerfeld parameter.

In the KEWPIE2 code, instead of the regular boundary condition imposed at the origin $(r \rightarrow 0)$, the incoming-wave boundary condition is adopted [83], under which the wave function can be expressed in the following form:

$$
u(r) \propto \sqrt{\frac{k}{k\left(r_{\mathrm{abs}}\right)}} \exp \left[-i \int_{r_{\mathrm{abs}}}^{r} k\left(r^{\prime}\right) \mathrm{d} r^{\prime}\right], \text { for } r \leq r_{\mathrm{abs}},
$$

where $r_{\text {abs }}$ refers to the absorption radius that is taken to be the bottom of the Coulomb pocket inside the barrier. Here, $k$ and $k(r)$ stand for the wave numbers associated with the energy $E_{\mathrm{cm}}$ and the local wave number for the $l$-th partial wave, respectively. They are given as follows:

$$
\begin{aligned}
k & =\sqrt{\frac{2 \mu E_{\mathrm{cm}}}{\hbar^{2}}}, \\
k(r) & =\sqrt{\frac{2 \mu}{\hbar^{2}}\left[E_{\mathrm{cm}}-V_{C}(r)-\mathfrak{R}\left[V_{\mathrm{opt}}(r)\right]-\frac{l(l+1) \hbar^{2}}{2 \mu r^{2}}\right]},
\end{aligned}
$$

where $\mathfrak{R}\left[V_{N}(r)\right]$ represents the real part of the optical potential (see Eq. (43)). It is known that the IWBC actually corresponds to the case where there is a strong absorption within the inner region of the potential, so that the incoming flux will never return back. All partial waves that penetrate through the barrier are thus absorbed.

To obtain the transmission coefficient, one needs to estimate the S-matrix. This can be done by matching the solutions of the Schrödinger equation to the Coulomb wave functions at a large distance $R_{\max }$. Finally, the $S$-matrix is expressed as

$$
S_{l}=\frac{H_{l}^{(-)}\left(\eta, k r_{0}\right) u\left(r_{1}\right)-H_{l}^{(-)}\left(\eta, k r_{1}\right) u\left(r_{0}\right)}{H_{l}^{(+)}\left(\eta, k r_{0}\right) u\left(r_{1}\right)-H_{l}^{(+)}\left(\eta, k r_{1}\right) u\left(r_{0}\right)}
$$


where $r_{0}$ and $r_{1}$ have been chosen to be $R_{\max }-\Delta r$ and $R_{\max }+\Delta r$, respectively, with $\Delta r$ being the radial mesh size required for the integration. The modified Numerov method has been employed for computing the radial wave functions $u\left(r_{0}\right)$ and $u\left(r_{1}\right)$ [12]. As for the two initial values within the modified Numerov method, they can be determined using the Runge-Kutta 4thorder method under the IWBC.

\section{Acknowledgements}

The authors acknowledge JSPS and the French-Japanese International Associated Laboratory for Nuclear Structure Problems (LIA-FJNSP) for partial support of this work. They greatly appreciate the warm hospitality and support provided by GANIL and RCNP at Osaka Univ., which enabled them to work together. H. L. is also indebted to Prof. Shen for helpful discussions and support. He would also like to thank the staff at Huzhou Univ. for their hospitality.

\section{References}

[1] M. Morjean, M. Chevallier, C. Cohen, D. Dauvergne, J. Dural, J. Galin, F. Goldenbaum, D. Jacquet, R. Kirsch, E. Lienard, B. Lott, A. Peghaire, Y. Perier, J. Poizat, G. Prevot, J. Remillieux, D. Schmaus, and M. Toulemonde, Nucl. Phys. A 630, 200 (1998).

[2] F. Goldenbaum, M. Morjean, J. Galin, E. Liénard, B. Lott, Y. Périer, M. Chevallier, D. Dauvergne, R. Kirsch, J. C. Poizat, J. Remillieux, C. Cohen, A. L'Hoir, G. Prévot, D. Schmaus, J. Dural, M. Toulemonde, and D. Jacquet, Phys. Rev. Lett. 82, 5012 (1999).

[3] M. Morjean, D. Jacquet, J. L. Charvet, A. L'Hoir, M. Laget, M. Parlog, A. Chbihi, M. Chevallier, C. Cohen, D. Dauvergne, R. Dayras, A. Drouart, C. Escano-Rodriguez, J. D. Frankland, R. Kirsch, P. Lautesse, L. Nalpas, C. Ray, C. Schmitt, C. Stodel, L. Tassan-Got, E. Testa, and C. Volant, Phys. Rev. Lett. 101, 072701 (2008).

[4] M. O. Frégeau, D. Jacquet, M. Morjean, E. Bonnet, A. Chbihi, J. D. Frankland, M. F. Rivet, L. Tassan-Got, F. Dechery, A. Drouart, L. Nalpas, X. Ledoux, M. Parlog, C. Ciortea, D. Dumitriu, D. Fluerasu, M. Gugiu, F. Gramegna, V. L. Kravchuk, T. Marchi, D. Fabris, A. Corsi, and S. Barlini, Phys. Rev. Lett. 108, 122701 (2012).

[5] B. Bouriquet, Y. Abe, and D. Boilley, Comput. Phys. Commun. 159, 1 (2004).

[6] A. Marchix, Etude de l'influence de l'énergie de correction de couches sur les réactions nucléaires menant à la région des noyaux superlourds, Thesis, Université de Caen (2007).

[7] C. Wang, J. Zhang, Z. Z. Ren, and C. W. Shen, Phys. Rev. C 82, 054605 (2010).

[8] J. Zhang, C. Wang, and Z. Ren, Nucl. Phys. A 909, 36 (2013).

[9] R. Ferrer, B. Bastin, D. Boilley, P. Creemers, P. Delahaye, E. Liénard, X. Fléchard, S. Franchoo, L. Ghys, M. Huyse, Y. Kudryavtsev, N. Lecesne, H. Lü, F. Lutton, E. Mogilevskiy, D. Pauwels, J. Piot, D. Radulov, L. Rens, H. Savajols, J. Thomas, E. Traykov, C. V. Beveren, P. V. den Bergh, and P. V. Duppen, Nucl. Instrum. Methods Phys. Res., Sect. B 317, Part B, 570 (2013).

[10] G. Henning, T. L. Khoo, A. Lopez-Martens, D. Seweryniak, M. Alcorta, M. Asai, B. B. Back, P. F. Bertone, D. Boilley, M. P. Carpenter, C. J. Chiara, P. Chowdhury, B. Gall, P. T. Greenlees, G. Gürdal, K. Hauschild, A. Heinz, C. R. Hoffman, R. V. F. Janssens, A. V. Karpov, B. P. Kay, F. G. Kondev, S. Lakshmi, T. Lauritsen, C. J. Lister, E. A. McCutchan, C. Nair, J. Piot, D. Potterveld, P. Reiter, A. M. Rogers, N. Rowley, and S. Zhu, Phys. Rev. Lett. 113, 262505 (2014).

[11] N. Bohr, Nature 137, 334 (1936).

[12] K. Hagino, N. Rowley, and A. Kruppa, Comput. Phys. Commun. 123, 143 (1999).

[13] G. Audi, M. Wang, A. Wapstra, F. Kondev, M. MacCormick, X. Xu, and B. Pfeiffer, Chin. Phys. C 36, 1287 (2012).
[14] M. Wang, G. Audi, A. Wapstra, F. Kondev, M. MacCormick, X. Xu, and B. Pfeiffer, Chin. Phys. C 36, 1603 (2012).

[15] P. Möller, J. Nix, W. Myers, and W. Swiatecki, At. Data. Nucl. Data Tables 59, 185 (1995).

[16] V. Weisskopf, Phys. Rev. 52, 295 (1937).

[17] V. F. Weisskopf and D. H. Ewing, Phys. Rev. 57, 472 (1940).

[18] W. Hauser and H. Feshbach, Phys. Rev. 87, 366 (1952).

[19] W. J. Świątecki, K. Siwek-Wilczyńska, and J. Wilczyński, Phys. Rev. C 71, 014602 (2005).

[20] W. Loveland, Phys. Rev. C 76, 014612 (2007).

[21] T. Cap, K. Siwek-Wilczyńska, and J. Wilczyński, Phys. Rev. C 83, 054602 (2011).

[22] A. Gavron, Phys. Rev. C 21, 230 (1980).

[23] R. Charity, M. McMahan, G. Wozniak, R. McDonald, L. Moretto, D. Sarantites, L. Sobotka, G. Guarino, A. Pantaleo, L. Fiore, A. Gobbi, and K. Hildenbrand, Nucl. Phys. A 483, 371 (1988).

[24] R. J. Charity, Phys. Rev. C 82, 014610 (2010).

[25] F. Pühlhofer, Nucl. Phys. A 280, 267 (1977).

[26] T. Thomas, Nucl. Phys. 53, 558 (1964).

[27] T. Thomas, Nucl. Phys. 53, 577 (1964).

[28] A. Cole, N. Longequeue, J. Menet, J. Lucas, R. Ost, and J. Viano, Nucl. Phys. A 341, 284 (1980).

[29] P. Young, E. D. Arthur, and M. Chadwick, "Comprehensive nuclear model calculations: Introduction to the theory and use of the gnash code," Tech. Rep. (Los Alamos National Lab., NM (United States), 1992).

[30] M. Herman, R. Capote, B. Carlson, P. Obložinský, M. Sin, A. Trkov, H. Wienke, and V. Zerkin, Nucl. Data Sheets 108, 2655 (2007), special Issue on Evaluations of Neutron Cross Sections.

[31] A. Koning, S. Hilaire, and M. Duijvestijn, in Proceedings of the International Conference on nuclear data for science and technology (2007) pp. 211-214.

[32] N. Bohr and J. A. Wheeler, Phys. Rev. 56, 426 (1939).

[33] H. Kramers, Physica 7, 284 (1940).

[34] P. Grangé and H. A. Weidenmüller, Phys. Lett. B 96, 26 (1980).

[35] P. Grangé, L. Jun-Qing, and H. A. Weidenmüller, Phys. Rev. C 27, 2063 (1983).

[36] H. A. Weidenmüller and Z. Jing-Shang, Phys. Rev. C 29, 879 (1984).

[37] Y. Abe, C. Grégoire, and H. Delagrange, Le Journal de Physique Colloques 47, C4 (1986).

[38] Y. Abe, S. Ayik, P.-G. Reinhard, and E. Suraud, Phys. Rep. 275, 49 (1996).

[39] A. Junghans, M. de Jong, H.-G. Clerc, A. Ignatyuk, G. Kudyaev, and K.-H. Schmidt, Nucl. Phys. A 629, 635 (1998).

[40] V. Plujko, Nucl. Phys. A 649, 209 (1999).

[41] V. Plujko, Acta Phys. Pol. B 31, 435 (2000).

[42] J. Blocki, Y. Boneh, J. R. Nix, J. Randrup, M. Robel, A. J. Sierk, and W. J. Swiatecki, Ann. Phys. 113, 330 (1978).

[43] W. Reisdorf, Z. Phys. A 300, 227 (1981).

[44] I. Dutt and R. K. Puri, Phys. Rev. C 81, 044615 (2010).

[45] W. D. Myers and W. J. Świaţecki, Phys. Rev. C 62, 044610 (2000).

[46] K. Siwek-Wilczyńska and J. Wilczyński, Phys. Rev. C 69, 024611 (2004).

[47] R. Bass, Phys. Rev. Lett. 39, 265 (1977).

[48] K. H. Schmidt and W. Morawek, Rep. Prog. Phys. 54, 949 (1991).

[49] A. Quint, W. Reisdorf, K.-H. Schmidt, P. Armbruster, F. Heßberger, S. Hofmann, J. Keller, G. Münzenberg, H. Stelzer, H.-G. Clerc, W. Morawek, and C.-C. Sahm, Z. Phys. A 346, 119 (1993).

[50] W. Swiatecki, Nucl. Phys. A 376, 275 (1982).

[51] G. Royer and B. Remaud, Nucl. Phys. A 444, 477 (1985).

[52] J. Blocki, H. Feldmeier, and W. Swiatecki, Nucl. Phys. A 459, 145 (1986).

[53] Y. Abe, D. Boilley, B. G. Giraud, and T. Wada, Phys. Rev. E 61, 1125 (2000).

[54] Y. Abe, Eur. Phys. J. A 13, 143 (2002).

[55] C. Shen, D. Boilley, Q. Li, J. Shen, and Y. Abe, Phys. Rev. C 83, 054620 (2011).

[56] W. Q. Shen, J. Albinski, R. Bock, A. Gobbi, S. Gralla, K. D. Hildenbrand, N. Herrmann, J. Kuzminski, W. F. J. Müller, H. Stelzer, J. Töke, B. B. Back, S. Bjørnholm, S. P. Sørensen, A. Olmi, and G. Guarino, Europhys. Lett. 1, 113 (1986). 
[57] B. B. Back, P. B. Fernandez, B. G. Glagola, D. Henderson, S. Kaufman, J. G. Keller, S. J. Sanders, F. Videbæk, T. F. Wang, and B. D. Wilkins, Phys. Rev. C 53, 1734 (1996).

[58] C. Shen, G. Kosenko, and Y. Abe, Phys. Rev. C 66, 061602 (2002).

[59] C. Shen, Y. Abe, D. Boilley, G. Kosenko, and E. Zhao, Int. J. Mod. Phys. E 17, 66 (2008)

[60] H. Bateman, in Proc. Cambridge Philos. Soc, Vol. 15 (1910) pp. 423 427.

[61] S. Hassani and P. Grangé, Phys. Lett. B 137, 281 (1984).

[62] P. Grangé, S. Hassani, H. A. Weidenmüller, A. Gavron, J. R. Nix, and A. J. Sierk, Phys. Rev. C 34, 209 (1986).

[63] C. R. Morton, A. C. Berriman, M. Dasgupta, D. J. Hinde, J. O. Newton, K. Hagino, and I. J. Thompson, Phys. Rev. C 60, 044608 (1999).

[64] V. Zagrebaev and V. Samarin, Phys. At. Nucl. 67, 1462 (2004).

[65] K. Hagino and N. Takigawa, Prog. Theor. Phys. 128, 1001 (2012).

[66] E. C. Kemble, Phys. Rev. 48, 549 (1935).

[67] J. Blocki, J. Randrup, W. Swiatecki, and C. Tsang, Ann. Phys. 105, 427 (1977).

[68] W. Reisdorf, F. Hessberger, K. Hildenbrand, S. Hofmann, G. Münzenbergnzenberg, K.-H. Schmidt, J. Schneider, W. Schneider, K. Sümmerer, G. Wirth, J. Kratz, and K. Schlitt, Nucl. Phys. A 438, 212 (1985)

[69] Y. Abe, D. Boilley, G. Kosenko, J. D. Bao, C. W. Shen, B. Giraud T. Wada, et al., Prog. Theor. Phys. Supp. 146, 104 (2002)

[70] D. Boilley, Y. Abe, and J.-D. Bao, Eur. Phys. J. A 18, 627 (2003), 10.1140/epja/i2003-10088-0.

[71] D. Boilley, H. Lü, C. Shen, Y. Abe, and B. G. Giraud, Phys. Rev. C 84, 054608 (2011).

[72] W. Świątecki, K. Siwek-Wilczyńska, and J. Wilczyński, Acta Phys. Polon. B 34, 2049 (2003).

[73] H. Delagrange, C. Grégoire, F. Scheuter, and Y. Abe, Z. Phys. A 323, 437 (1986).

[74] I. Dostrovsky, Z. Fraenkel, and G. Friedlander, Phys. Rev. 116, 683 (1959).

[75] M. A. McMahan and J. M. Alexander, Phys. Rev. C 21, 1261 (1980).

[76] F. D. Becchetti and G. W. Greenlees, Phys. Rev. 182, 1190 (1969).

[77] D. Wilmore and P. Hodgson, Nucl. Phys. 55, 673 (1964).

[78] R. Varner, W. Thompson, T. McAbee, E. Ludwig, and T. Clegg, Phys. Rep. 201, 57 (1991)

[79] A. Koning and J. Delaroche, Nucl. Phys. A 713, 231 (2003).

[80] M. Nolte, H. Machner, and J. Bojowald, Phys. Rev. C 36, 1312 (1987).

[81] V. Avrigeanu, P. E. Hodgson, and M. Avrigeanu, Phys. Rev. C 49, 2136 (1994).

[82] J. M. Alexander, M. T. Magda, and S. Landowne, Phys. Rev. C 42, 1092 (1990).

[83] G. Rawitscher, Nucl. Phys. 85, 337 (1966).

[84] P. Reiter, T. L. Khoo, T. Lauritsen, C. J. Lister, D. Seweryniak, A. A. Sonzogni, I. Ahmad, N. Amzal, P. Bhattacharyya, P. A. Butler, M. P. Carpenter, A. J. Chewter, J. A. Cizewski, C. N. Davids, K. Y. Ding, N. Fotiades, J. P. Greene, P. T. Greenlees, A. Heinz, W. F. Henning, R.D. Herzberg, R. V. F. Janssens, G. D. Jones, F. G. Kondev, W. Korten, M. Leino, S. Siem, J. Uusitalo, K. Vetter, and I. Wiedenhöver, Phys. Rev. Lett. 84, 3542 (2000)

[85] V. F. Weisskopf, Phys. Rev. 83, 1073 (1951).

[86] D. M. Brink, unpublished, Ph.D. thesis, Oxford University (1955)

[87] P. Axel, Phys. Rev. 126, 671 (1962).

[88] J. Kopecky, M. Uhl, and R. E. Chrien, Phys. Rev. C 47, 312 (1993).

[89] R. Capote, M. Herman, P. Obložinský, P. Young, S. Goriely, T. Belgya, A. Ignatyuk, A. Koning, S. Hilaire, V. Plujko, M. Avrigeanu, O. Bersillon, M. Chadwick, T. Fukahori, Z. Ge, Y. Han, S. Kailas, J. Kopecky, V. Maslov, G. Reffo, M. Sin, E. Soukhovitskii, and P. Talou, Nucl. Data Sheets 110, 3107 (2009), special Issue on Nuclear Reaction Data.

[90] P. Ring, L. Robledo, J. Egido, and M. Faber, Nucl. Phys. A 419, 261 (1984).

[91] J. L. Egido and H. A. Weidenmüller, Phys. Rev. C 39, 2398 (1989).

[92] J. L. Egido and P. Ring, J. Phys. G 19, 1 (1993).

[93] V. Plujko, O. Gorbachenko, and E. Kulich, Int. J. Mod. Phys. E 18, 996 (2009).

[94] V. Plujko, R. Capote, and O. Gorbachenko, At. Data. Nucl. Data Tables 97, 567 (2011).

[95] B. L. Berman and S. C. Fultz, Rev. Mod. Phys. 47, 713 (1975).

[96] D. L. Hill and J. A. Wheeler, Phys. Rev. 89, 1102 (1953).
[97] V. Strutinsky, Phys. Lett. B 47, 121 (1973).

[98] W. Myers and W. Swiatecki, Nucl. Phys. A 601, 141 (1996).

[99] A. Dobrowolski, K. Pomorski, and J. Bartel, Phys. Rev. C 75, 024613 (2007).

[100] N. Wang, Z. Liang, M. Liu, and X. Wu, Phys. Rev. C 82, 044304 (2010). [101] M. Liu, N. Wang, Y. Deng, and X. Wu, Phys. Rev. C 84, 014333 (2011).

[102] N. Chamel, S. Goriely, and J. Pearson, Nucl. Phys. A 812, 72 (2008).

[103] S. Goriely, N. Chamel, and J. M. Pearson, Phys. Rev. Lett. 102, 152503 (2009).

[104] W. D. Myers and W. J. Światecki, Phys. Rev. C 60, 014606 (1999).

[105] F. A. Ivanyuk and K. Pomorski, Phys. Rev. C 79, 054327 (2009).

[106] M. Grossjean and H. Feldmeier, Nucl. Phys. A 444, 113 (1985).

[107] A. Gilbert and A. G. W. Cameron, Can. J. Phys. 43, 1446 (1965).

[108] J. Tōke and W. Światecki, Nucl. Phys. A 372, 141 (1981).

[109] R. W. Hasse and W. D. Myers, Geometrical relationships of macroscopic nuclear physics (Springer-Verlag Heidelberg, 1988)

[110] W. J. Światecki, Phys. Rev. 104, 993 (1956).

[111] M. Dahlinger, D. Vermeulen, and K.-H. Schmidt, Nucl. Phys. A 376, 94 (1982).

[112] B. Nerlo-Pomorska, K. Pomorski, and J. Bartel, Phys. Rev. C 74, 034327 (2006)

[113] A. Iljinov, M. Mebel, N. Bianchi, E. D. Sanctis, C. Guaraldo, V. Lucherini, V. Muccifora, E. Polli, A. Reolon, and P. Rossi, Nucl. Phys. A 543, 517 (1992).

[114] A. Bohr and B. R. Mottelson, Nuclear structure, vol. II (Benjamin, New York, 1975)

[115] A. V. Ignatyuk, G. Smirenkin, and A. Tishin, Yad. Fiz., v. 21, no. 3, pp. 485-490 21 (1975).

[116] V. I. Zagrebaev, Y. Aritomo, M. G. Itkis, Y. T. Oganessian, and M. Ohta, Phys. Rev. C 65, 014607 (2001).

[117] A. Bohr and B. R. Mottelson, Nuclear structure, vol. I (W. A. Benjmain, Inc., 1969).

[118] D. Hilscher and H. Rossner, Ann. Phys. Fr. 17, 471 (1992).

[119] P. C. Rout, D. R. Chakrabarty, V. M. Datar, S. Kumar, E. T. Mirgule, A. Mitra, V. Nanal, S. P. Behera, and V. Singh, Phys. Rev. Lett. 110, 062501 (2013)

[120] P. Möller, A. J. Sierk, T. Ichikawa, A. Iwamoto, R. Bengtsson, H. Uhrenholt, and S. Åberg, Phys. Rev. C 79, 064304 (2009).

[121] R. Sagaidak, Proceedings of the VI International School-Seminar on Heavy Ion Physics, 22-27 September 1997, Dubna, Russia (1998).

[122] R. Yanez, W. Loveland, L. Yao, J. Barrett, S. Zhu, B. Back, T. Khoo, M. Alcorta, and M. Albers, Phys. Rev. Lett. 112, 152702 (2014).

[123] J. Tōke, R. Bock, G. Dai, A. Gobbi, S. Gralla, K. Hildenbrand, J. Kuźminski, W. Müller, A. Olmi, W. Reisdorf, S. Bjørnholm, and B. Back, Phys. Lett. B 142, 258 (1984).

[124] J. Tōke, R. Bock, G. Dai, A. Gobbi, S. Gralla, K. Hildenbrand, J. Kuzminski, W. Müller, A. Olmi, H. Stelzer, B. Back, and S. Bjørnholm, Nucl. Phys. A 440, 327 (1985).

[125] W. Q. Shen, J. Albinski, A. Gobbi, S. Gralla, K. D. Hildenbrand, N. Herrmann, J. Kuzminski, W. F. J. Müller, H. Stelzer, J. Tke, B. B. Back, S. Bjrnholm, and S. P. Srensen, Phys. Rev. C 36, 115 (1987).

[126] K. Nishio, H. Ikezoe, Y. Nagame, M. Asai, K. Tsukada, S. Mitsuoka, K. Tsuruta, K. Satou, C. J. Lin, and T. Ohsawa, Phys. Rev. Lett. 93 , 162701 (2004)

[127] T. Editors, Phys. Rev. A 83, 040001 (2011).

[128] D. Morrissey, W. Loveland, R. Otto, and G. Seaborg, Phys. Lett. B 74, 35 (1978)

[129] A. J. Pacheco, J. O. Fernández Niello, D. E. DiGregorio, M. di Tada, J. E. Testoni, Y. Chan, E. Chávez, S. Gazes, E. Plagnol, and R. G. Stokstad, Phys. Rev. C 45, 2861 (1992).

[130] E. Prokhorova, A. Bogachev, M. Itkis, I. Itkis, G. Knyazheva, N. Kondratiev, E. Kozulin, L. Krupa, Y. Oganessian, I. Pokrovsky, V. Pashkevich, and A. Rusanov, Nucl. Phys. A 802, 45 (2008).

[131] Y. T. Oganessian, V. K. Utyonkov, Y. V. Lobanov, F. S. Abdullin, A. N. Polyakov, I. V. Shirokovsky, Y. S. Tsyganov, A. N. Mezentsev, S. Iliev, V. G. Subbotin, A. M. Sukhov, K. Subotic, O. V. Ivanov, A. N. Voinov, V. I. Zagrebaev, K. J. Moody, J. F. Wild, N. J. Stoyer, M. A. Stoyer, and R. W. Lougheed, Phys. Rev. C 64, 054606 (2001).

[132] H. Gäggeler, D. Jost, A. Türler, P. Armbruster, W. Brüchle, H. Folger, F. Heßberger, S. Hofmann, G. Münzenberg, V. Ninov, W. Reisdorf, M. Schädel, K. Sümmerer, J. Kratz, U. Scherer, and M. Leino, Nucl. 
Phys. A 502, 561 (1989).

[133] G. Henning, Stability of Transfermium Elements at High Spin : Measuring the Fission Barrier of ${ }^{254}$ No, Thesis, Université Paris Sud - Paris XI (2012).

[134] M. Abramowitz and I. A. Stegun, Handbook of mathematical functions: with formulas, graphs, and mathematical tables, 55 (Courier Dover Publications, 1972). 